\title{
Identification of long noncoding RNA RP11-89K21.1 and RP11-357H14.17 as prognostic signature of endometrial carcinoma via integrated bioinformatics analysis
}

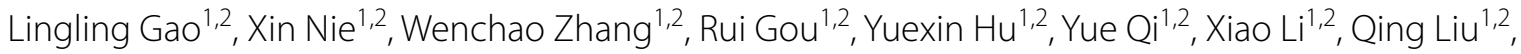
Juanjuan Liu ${ }^{1,2}$ and Bei $\operatorname{Lin}^{1,2^{*}}$ (D)

\begin{abstract}
Background: Endometrial carcinoma (EC) is one of the most common malignant tumors in gynecology. The potential functions and mechanisms of long noncoding RNAs (IncRNAs) in the occurrence and progression of EC remains unclear. It's meaningful to explore IncRNAs signature for providing prognostic value of EC.
\end{abstract}

Methods: The differentially expressed IncRNAs and their prognostic values in EC were investigated based on The Cancer Genome Atlas (TCGA) database; the transcriptional factors (TFs), the competing endogenous RNA (ceRNA) mechanism, functional regulatory network and immune infiltration of RP11-89K21.1 and RP11-357H14.17 were further explored by various bioinformatics tools and databases.

Results: We firstly identified high expression of RP11-89K21.1 and RP11-357H14.17 were closely associated with shorten overall survival (OS) and poor prognosis in patients with EC. We also elucidated the networks of transcription factor and co-expression genes associated with RP11-89K21.1 and RP11-357H14.17. Furthermore, the ceRNA network mechanism was successfully constructed through 2 IncRNAs (RP11-89K21.1 and RP11-357H14.17), 11 miRNAs and 183 mRNAs. Functional enrichment analysis revealed that the targeting genes of RP11-89K21.1 and RP11-357H14.17 were strongly associated with microRNAs in cancer, vessel development, growth regulation, growth factor and cell differentiation, and involved in pathways including pathways in cancer, microRNAs in cancer and apoptotic signaling pathway.

Conclusions: We demonstrated for the first time that RP11-89K21.1 and RP11-357H14.17 may play crucial roles in the occurrence, development and malignant biological behavior of EC, and can be regarded as potential prognostic biomarkers for EC.

Keywords: Endometrial carcinoma, Long noncoding RNA, Prognosis, Competing endogenous RNA, Bioinformatics analysis

\footnotetext{
*Correspondence: linbei88@hotmail.com

${ }^{2}$ Key Laboratory of Maternal-Fetal Medicine of Liaoning Province, Key

Laboratory of Obstetrics and Gynecology of Higher Education of Liaoning Province, Liaoning, China

Full list of author information is available at the end of the article
}

\section{Background}

Endometrial carcinoma (EC) is one of the most common types of malignancies in the female reproductive system, accounting for $20 \%$ to $30 \%$ of the total number of malignant tumors in female genital tract. In some developed countries, the incidence of EC is higher than that

(c) The Author(s) 2020. This article is licensed under a Creative Commons Attribution 4.0 International License, which permits use, sharing, adaptation, distribution and reproduction in any medium or format, as long as you give appropriate credit to the original author(s) and the source, provide a link to the Creative Commons licence, and indicate if changes were made. The images or other third party material in this article are included in the article's Creative Commons licence, unless indicated otherwise in a credit line to the material. If material is not included in the article's Creative Commons licence and your intended use is not permitted by statutory regulation or exceeds the permitted use, you will need to obtain permission directly from the copyright holder. To view a copy of this licence, visit http://creativeco mmons.org/licenses/by/4.0/. The Creative Commons Public Domain Dedication waiver (http://creativecommons.org/publicdomain/ zero/1.0/) applies to the data made available in this article, unless otherwise stated in a credit line to the data. 
of cervical cancer, which ranks first among gynecological tumors [1]. In recent years, the incidence of EC in China has increased year by year and ranked second of gynecological cancer, showing younger trend and severely threatening the physical and mental health of women [2]. At present, the treatment of endometrial cancer included surgery, radiotherapy, and chemotherapy, while patients with advanced EC may have distant metastasis, postoperative recurrence, and poor prognosis. Therefore, exploring potential biomarkers closely associated with the occurrence and development of EC are of great value for early diagnosis and targeted therapy of endometrial carcinoma.

Long noncoding RNAs (lncRNAs) are a group of noncoding RNAs with more than $200 \mathrm{bp}$ in length with no or limited protein-coding function, which were first discovered in mice in 2002 and lack of specific and complete open reading frame [3]. LncRNAs, as important regulators of transcription and translation, have been found not only involved in physiological and pathological processes, including chromatin remodeling, transcription, post-transcriptional translation, cell proliferation, differentiation and metabolic reprogramming $[4,5]$, but also playing a pivotal role in the occurrence and development of malignant tumors [6]. Abnormal expression of lncRNAs can affect the development and progression of many kinds of malignant tumors, such as prostate cancer [7], ovarian cancer [8], breast cancer [9] and gastric cancer [10]. In recent years, a variety of IncRNAs have been identified to be essential for the initiation, progression and malignant behaviors of endometrial carcinoma [11]. High expression of MALAT1 [12], HOTAIR [13] and NEAT1 [14] were closely associated with the poor prognosis of EC and promoted the proliferation, metastasis and EMT of endometrial cancer cells. Other studies have shown that the expression of MEG3 [15] and FER1L4 [16] were decreased in EC, and high expression of MEG3 and FER1L4 inhibited the proliferation, migration and invasion of endometrial cancer cells. These studies suggest that lncRNAs play a crucial role in the prognosis and malignant biological behaviors of EC.

In this study, we investigated the differentially expressed lncRNAs in EC based on the Cancer Genome Atlas (TCGA) database and identified two lncRNA RP11-89K21.1 and RP11-357H14.17 and their correlation with the occurrence, development, prognostic value and functional regulatory network of EC. We also explored the upstream transcriptional regulatory factors, co-expression genes and binding proteins of lncRNAs and their relationship with immune infiltration. Furthermore, we explored their potential roles and molecular mechanisms in EC utilizing competing endogenous RNA (ceRNA) (lncRNA-miRNA-mRNA) hypothesis, which is extremely meaningful to provide a new strategy for early diagnosis and treatment of endometrial carcinoma.

\section{Materials and methods \\ Screening for differentially expressed IncRNAs by circIncRNAnet}

CirclncRNAnet (http://120.126.1.61/circlnc/index.php) [17] is an online tool for exploring lncRNA and circRNA chip or sequencing expression data integrated with more than 20 tumor types in TCGA database, which included several analysis modules, such as heat map, box diagram, co-expression scatter map, circos map, gene functional enrichment analysis, RBP-RNA binding protein network and miRNA network. We explored the differentially expressed lncRNAs in EC based on Uterine Corpus Endometrial Carcinoma (UCEC) with circlncRNAnet, and constructed Circos map and heat map of LncRNAs co-expression genes, Pearson's correlation analysis was employed for exhibiting significant correlation between co-expressed genes and these lncRNAs (default: $|\mathrm{r}|>0.5$ ). A total of 10,978 lncRNAs were identified in the cohort and we obtained 121 dysregulated lncRNAs (77 upregulated and 44 downregulated).The screening criteria were defined as follows: $\mid$ Log2 fold change $\mid>4$ and $P<0.01$.

\section{The prognosis of dysregulated IncRNAs analyzed with GEPIA and Kaplan-Meier plotter}

GEPIA (http://gepia.cancer-pku.cn/) [18] is a newly webbased tool that contains sequencing expression data from 9736 tumor samples of 33 cancer types and 8587 normal samples. The database includes a variety of analysis modules such as differential gene expression analysis, survival and prognosis analysis, correlation analysis, as well as dimensionality reduction analysis. In this study, GEPIA database was employed to further analyze the expressionand prognostic value of differentially expressed lncRNAs in UCEC. The expression analysis of these genes performed by one-way ANOVA, and the filter criteria were as follows: $|\log 2 \mathrm{FC}|>1, P$ value $<0.05$, "median", Hazards Ratio (HR) and 95\% confidence interval. The Kaplan-Meier (KM) Plotter (http://kmplot.com) [19] is an effective tool for detecting the prognosis of patients with tumors. According to the expression of lncRNAs, patients with EC were divided into two groups: high and low expression group. The hazard ratio (HR) at a 95\% confidence interval and log-rank $P$-values were also investigated online. The filter conditions were as follows: cancer: pan-cancer RNA-seq (Uterus corpus endometrial carcinoma); survival: overall survival (OS); follow-up threshold: 120 months. 


\section{The cellular localization of IncRNAs}

UCSC (https://genome-asia.ucsc.eduk/index.html) [20] provides a web-based interface that helps users browse gene information, view genome annotation assemblies and download gene sequences. LNCipedia (https://ncip edia.org) [21] is a freely available annotated database of human lncRNAs transcriptional sequences and structures, which utilizes secondary structure information to establish a standard and unified classification and naming system. The tool offers insights into functions of over 1500 human lncRNAs, including evaluating coding ability, predicting open reading frame and secondary structure. LncLocator (https://LncLocatorwww.csbio.sjtu.edu. $\mathrm{cn} /$ bioinf/lncLocator/) [22] is a free public platform to predict the subcellular localization of lncRNAs based on a stacked ensemble classifier. Only by utilizing lncRNA sequence information, the distribution proportion of lncRNA in 5 subcellular localizations, including cytoplasm, nucleus, ribosome, cytosol and exosome, can be quickly obtained. In this study, lncRNAs sequences information were detected by UCSC and LNCipedia databases, and the cellular localization of lncRNAs were then determined by LncLocator.

\section{Prediction and expression of candidate miRNA with AnnoLnc and starBase database}

AnnoLnc (http://annolnc.cbi.pku.edu.cn) [23] is a web interface to systematically annotate newly identified human lncRNAs based on more than 700 data sources. The systematic annotation of lncRNAs cover a wide range of functions, including genome location, secondary structure, expression pattern, transcriptional regulation, miRNA interaction, protein interaction, genetic association and evolution. StarBase (http://starbase.sysu. edu.cn/) [24] provides an widely-used open-source platform for exploring ncRNA interactions based on 10,882 RNA sequence and 10,546 miRNA sequence of 32 cancer types, the platform can be used to perform the survival and differential expression analysis of miRNAs and lncRNAs. We predicted lncRNAs-binding miRNAs with AnnoLnc and further explored the expression of miRNAs in UCEC by starBase.

\section{Protein-protein interaction network and transcriptional regulatory network}

GeneMANIA (http://www.genemania.org) [25] is a flexible and user-friendly platform that can predict gene function, analyze gene lists and sequence genes with function assays, which provides three main cases: single gene queries, multiple gene queries and network search. The online tool can be used to construct protein-protein interaction (PPI) network and protein-DNA interaction, investigate potential signal pathway, gene and protein expression and protein domains. We explored lncRNArelated proteins and transcriptional regulatory molecules with AnnoLnc, and visualized the functions and regulatory networks of these molecules using GeneMANIA.

\section{Construction of IncRNA-miRNA-mRNA regulatory network} miRTarBase (http://mirtarbase.mbc.nctu.edu.tw/php/ index.php) [26] is a popular web interface which mainly collects miRNA target genes verified by different validation experiments and provides supportive evidences with literatures or assays. The database can be queried through different categories, such as miRNA, gene, disease, pathway and so on. Cytoscape [27] is a very powerful software for visualizing and analyzing network data, which allows users to construct many complex biological networks. Node and edge are the two core elements in the network diagram constructed by Cytoscape. miRNA target genes were explored by miRTarBase, and only those genes verified by at least one powerful experimental method were identified as miRNAs targets (reporter assay, Western blot or quantitative reverse transcription PCR). Cytoscape 3.7.1 was further employed to construct competing endogenous RNA (ceRNA) network (lncRNA-miRNA-mRNA).

\section{Functional enrichment analysis with Metascape}

Metascape (http://metascape.org) [28] is a user-friendly and effective tool for comprehensively annotating and analyzing single or multiple genes lists, which integrates many authoritative database resources such as Gene Ontology (GO), Kyoto Encyclopedia of Genes and Genomes (KEGG), UniProt and Drugbank. We can not only complete pathway enrichment and biological process annotation, but also construct protein-protein interaction (PPI) networks with Metascape. In this study, Metascape was used to analyze the GO and KEGG enrichment of differentially expressed genes related to lncRNAs. Restrictions: $P<0.01$, a minimum count of 3 , enrichment factor $>1.5$ were considered to be statistically significant. The PPI enrichment analysis in Metascape was based on the following databases: BioGrid, InWeb_IM and OmniPath. In addition, Molecular Complex Detection (MCODE) algorithm is applied to mine molecules with deeper network regulation relationships.

\section{Correlation between IncRNAs and immune infiltration analyzed with ImmLnc}

ImmLnc (http://bio-bigdata.hrbmu.edu.cn/ImmLnc) [29] is an online analysis website for investigating the immune-related function of lncRNAs across 33 cancer types with high-throughput methods, Users can investigated the lncRNA-pathways, lncRNA-immune cell type's 
correlation, and cancer-related lncRNAs. The ImmLnc serves as a valuable resource for exploring the lncRNA function and to further advance the identification of immunotherapy targets. In this study, we explored the correlation between lncRNAs and immune cell infiltration with ImmLnc.

\section{Results}

Identification of differentially expressed IncRNAs in Uterine Corpus Endometrial Carcinoma (UCEC) with circlncRNAnet and GEPIA

To investigated the roles of lncRNAs in the tumorigenesis, development of UCEC, we firstly identified differentially expressed lncRNAs in the LncRNA-TCGA module of cirlncRNAnet. A total of 10,978 lncRNAs were detected in Uterine Corpus Endometrial Carcinoma (UCEC), of which 121 lncRNAs were dysregulated (77 up-regulated and 44 down-regulated) (|Log2 fold change $\mid>4$ and $P<0.01$ ) (Additional file 1: Table S1). We further verified the expression of 121 differentially expressed lncRNAs in UCEC using the GEPIA and found that only RP11-89K21.1 and RP11-357H14.17 were significantly overexpressed in UCEC $(P<0.05)$ (Fig. 1a, b), consistent with the results from cirlncRNAnet. The expression of CTD-2314B22 0.1, CTD-2377D24.6, RP11$657 \mathrm{O} 9.1$ and LINC00668 were higher in UCEC than those in normal tissues, but the differences were not statistically significant $(P>0.05)$ (Fig. 1c-f). The expression of AP000892.6, ACTA2-AS1 and RP11-867G23.10 was significantly decreased in UCEC compared with normal tissues $(P<0.05)($ Fig. $1 \mathrm{~h}-\mathrm{i})$.

\section{Prognostic values of dysregulated IncRNAs including RP11-89K21.1 and RP11-357H14.17 in UCEC analyzed by GEPIA}

We further explored the prognostic values of the 121 differentially expressed lncRNAs in UCEC using GEPIA. The results showed that high expressions of RP11-89K21.1, RP11-357H14.17， CTD-2314B22.1， CTD-2377D24.6, RP11-657O9.1 and LINC00668 were significantly correlated with shortened overall survival (OS) in UCEC $(P=0.0088, P=0.025, P=0.026, P=0.0013, P=0.021$, and $P=0.0086$, respectively) (Fig. $2 \mathrm{a}-\mathrm{f}$, Table 1 ). Additionally, the expressions of AP000892.6, ACTA2-AS1 and RP11-867G23.10 were decreased in UCEC, while high expressions of AP000892.6, ACTA2-AS1 and RP11867G23.10 were significantly associated with poor prognosis (all $P<0.05$ ) (Fig. $2 g-i$ ). Kaplan-Meier plotter showed that high expressions of RP11-89K21.1 (Additional file 1: Figure S1a), LINC00668 (Additional file 1: Figure S1d) and ACTA2-AS1 (Additional file 1: Figure S1e) were also correlated with OS in UCEC (Additional

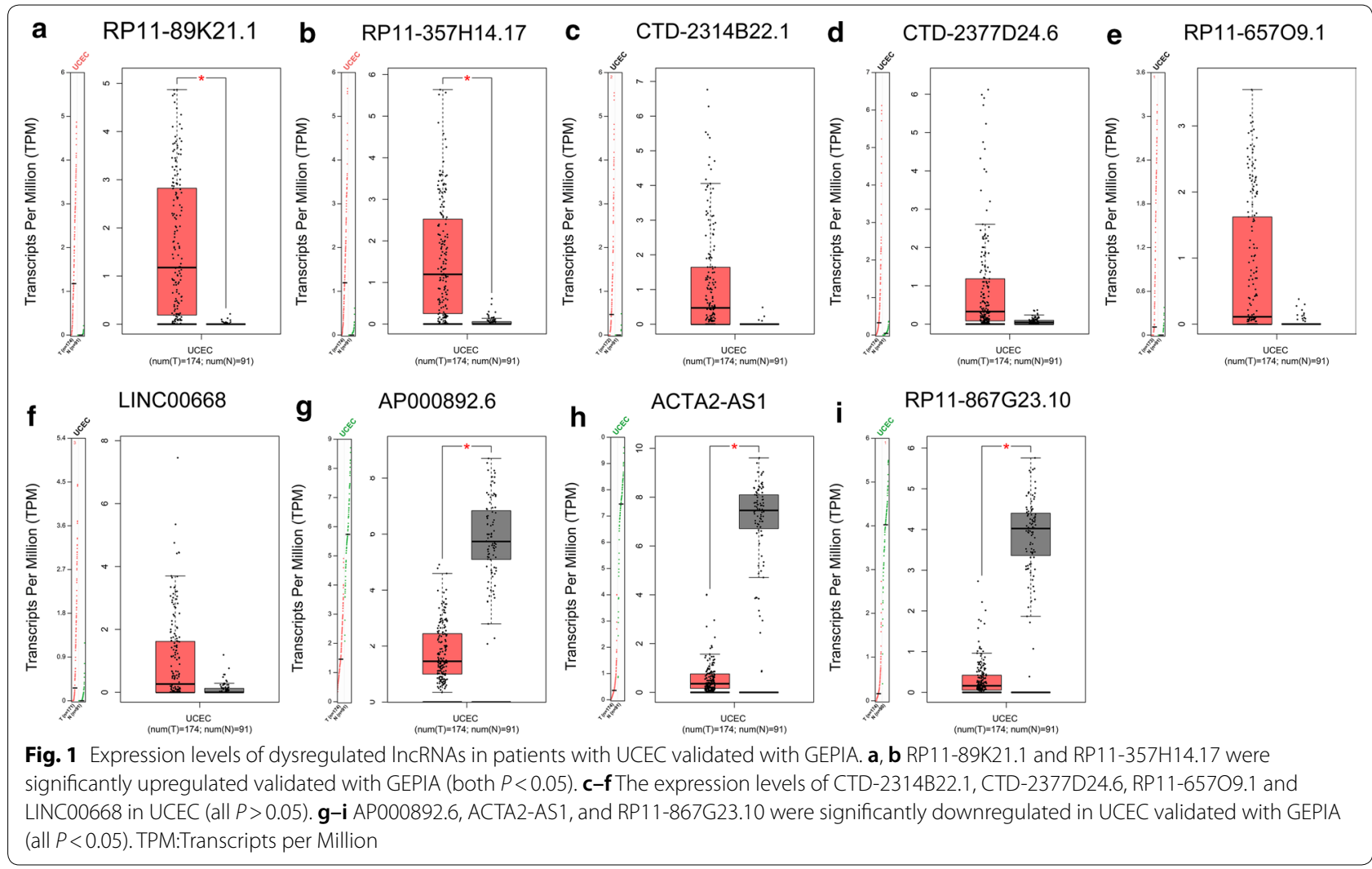



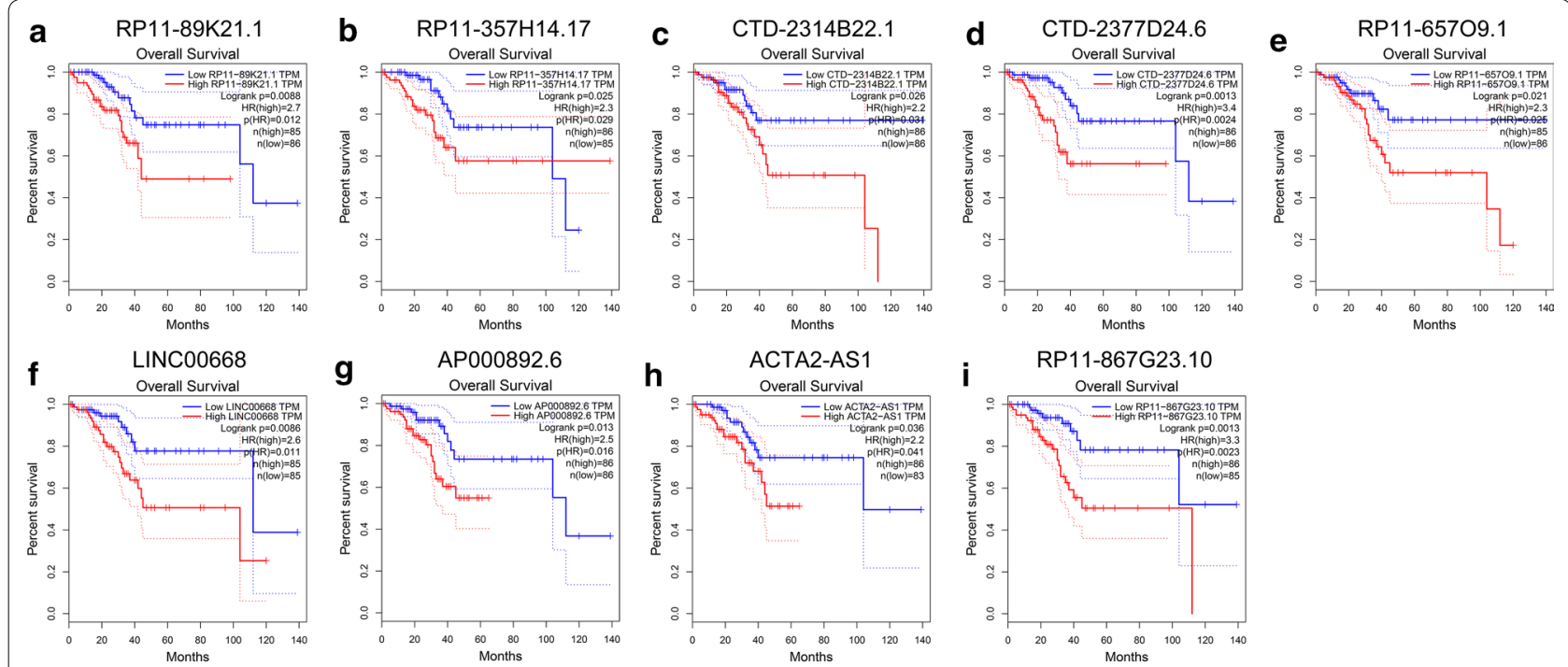

Fig. 2 Prognostic values of dysregulated IncRNAs including RP11-89K21.1 and RP11-357H14.17 in UCEC analyzed by GEPIA. a-i Relationship between RP11-89K21.1 (a), RP11-357H14.17 (b), CTD-2314B22.1 (c), CTD-2377D24.6 (d), RP11-65709.1 (e), LINC00668 (f), AP000892.6 (g), ACTA2-AS1 (h), RP11-867G23.10 (i) and overall survival (OS) of patients with UCEC

Table 1 The expression levels and prognostic values of differentially expressed IncRNAs including RP11-89K21.1 and RP11-357H14.17 in UCEC

\begin{tabular}{llllll}
\hline Gene & Ensembl ID & Genome location & Log2 fold change & P adj & Overall survival \\
\hline RP11-89K21.1 & ENSG00000259439 & chr2:44921077-44939199 & 7.113667606 & $3.31 \mathrm{E}-32$ & 0.0088 \\
RP11-357H14.17 & ENSG00000272763 & chr17:48635923-48647023 & 5.64219224 & $3.26 \mathrm{E}-28$ & 0.025 \\
CTD-2314B22.1 & ENSG00000258314 & chr14:19054341-19055551 & 5.415117641 & $5.14 \mathrm{E}-11$ & 0.026 \\
CTD-2377D24.6 & ENSG00000244649 & chr17:48705203-48707346 & 5.381493576 & $4.72 \mathrm{E}-21$ & 0.0013 \\
RP11-65709.1 & ENSG00000240086 & chr3:135373795-135439822 & 4.629555176 & $1.49 \mathrm{E}-09$ & 0.021 \\
LINC00668 & ENSG00000265933 & chr18:6925478-6928572 & 4.089425104 & $1.05 \mathrm{E}-11$ & 0.0086 \\
AP000892.6 & ENSG00000280143 & chr11:117204967-117210292 & -4.08259009 & $1.28 \mathrm{E}-36$ & 0.013 \\
ACTA2-AS1 & ENSG00000180139 & chr10:88932390-88933838 & -4.164362472 & $2.49 \mathrm{E}-36$ & 0.036 \\
RP11-867G23.10 & ENSG00000254510 & chr11:66409194-66417137 & -4.737563254 & $1.01 \mathrm{E}-29$ & 0.0013 \\
\hline
\end{tabular}

file 1: Table S1), RP11-357H14.17, CTD-2377D24.6 and AP000892.6 were not detected in Kaplan-Meier plotter. The above results suggest that high expression of RP1189K21.1 and RP11-357H14.17 may play important roles in the occurrence, development and prognosis of UCEC.

\section{The cellular location and co-expressed genes of RP11-89K21.1 and RP11-357H14.17}

The cellular localization of lncRNAs played crucial roles in their functions and molecular mechanisms, we explored the subcellular localizations of RP11-89K21.1 and RP11-357H14.17 with lncLocator. The results showed that RP11-89K21.17 was mainly located in cytosol and cytoplasm (score: 0.56 and 0.28 , respectively), RP11$357 \mathrm{H} 14.17$ was mainly located in cytosol and ribosome (score: 0.37 and 0.32 , respectively) (Fig. 3a). Therefore, it was more likely that RP11-89K21.1 and RP11-357H14.17 exerted their biological functions and potential mechanisms through the ceRNA network. We further explored the co-expression gene of RP11-89K21.1 and RP11$357 \mathrm{H} 14.17$ and visualized by Circos map and heat map with circlncRNAnet. The circos maps showed that chromosome distribution of the top 50 co-expressed genes associated with RP11-89K21.1 and RP11-357H14.17, which were mainly localized in the autosomes. There was no particular enrichment in chromosome 2 (where RP11-89K21.1 locates) and 7 (where RP11-357H14.17 locates). The heat map indicated that AC012354.6, RP1189K21.2, SIX3, SIX3-AS1, and Six3os1_1/2/4/5 were the co-expression gene of RP11-89K21.1, CTD-2377D24.6, 


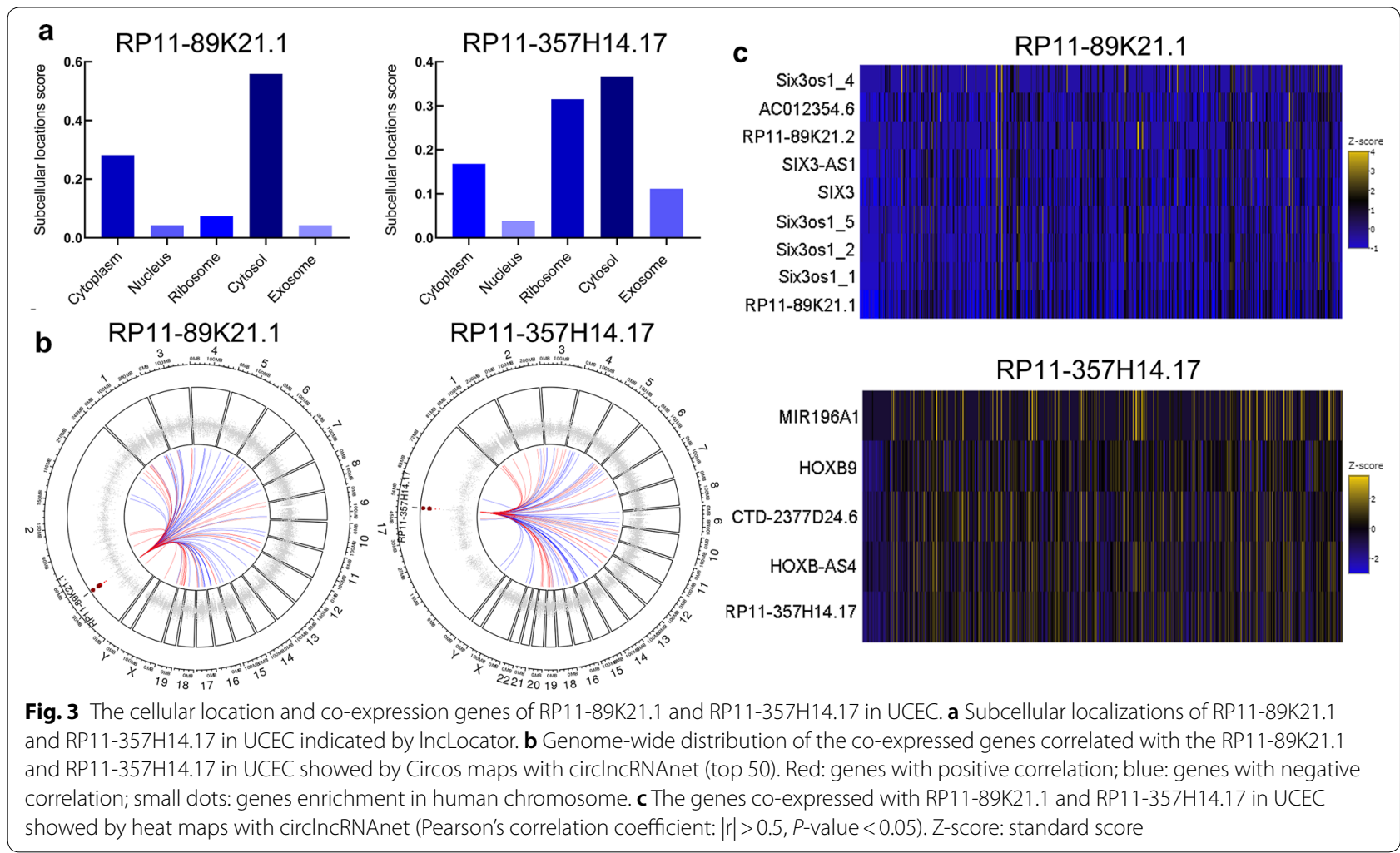

HOXB-AS4, HOXB9 and MIR196A1 were the co-expression gene of RP11-357H14.17 (all $P<0.05$ ) (Fig. 3b, c, Additional files 2, 3).

\section{Transcriptional regulation and protein interaction of RP11-89K21.1 and RP11-357H14.17}

Furthermore, we explored the transcriptional factors (TFs) and binding proteins of RP11-89K21.1 and RP11-357H14.17 by AnnoLnc. 31 TFs and 42 TFs were identified to be correlated with RP11-89K21.1 and RP11$357 \mathrm{H} 14.17$, respectively. 21 TFs of these two lncRNAs were commonly existed in the database (CEBPB, CHD1, c-Myc, CtBP2, CTCF, Egr-1, EZH2, GABP, Max, NRSF, p300, Pol2, Pol2-4H8, PU.1, Rad21, RBBP5, SUZ12, TCF7L2, YY1, Znf143, ZNF263) (Fig. 4a). We found that the expression of EZH2 in UCEC was significantly overexpressed, while the expression of TCF7L2 in UCEC was significantly decreased (both $P<0.05$ ). There was no significant difference in the expression levels of other TFs in UCEC $(P>0.05)$ (Fig. 4b). We further investigated that EZH2 expression was positively correlated with both RP11-89K21.1 and RP11-357H14.17 using starBase $(r=0.118, P=5.67 \mathrm{e}-03$ and $\mathrm{r}=0.103, P=1.57 \mathrm{e}-02$, respectively) (Fig. 4c). Based on the correlation coefficients were too small, we further explored the correlation between EZH2 and RP11-89K21.1, RP11-357H14.17 using GEPIA, the result showed that the expression of EZH2 was positively correlated with both RP1189K21.1 $(\mathrm{R}=0.3, \quad P=8.8 \mathrm{e}-07)$ and $\mathrm{RP} 11-357 \mathrm{H} 14.17$ $(\mathrm{R}=0.31, P=3.7 \mathrm{e}-07$ ) (Fig. $4 \mathrm{~d}$ ). What's more, the binding proteins of RP11-89K21.1 included CBWD7, GUSB, ABRA, MTUS2, VAMP4 (top 5) (Fig. 4e), and the binding proteins of RP11-357H14.17 included CLIC1, CNN2, TCTN1, TMEM240, ZNF836 (top 5) (Fig. 4f). The networks of the transcription factors (Fig. 4e) and binding proteins (Fig. 4f) were visualized through GeneMANIA (Additional files: 4, 5, 6, 7).

\section{Construction of IncRNA-miRNA-mRNA network}

The subcellular locations of lncRNAs were closely correlated with their potential functions and mechanisms in tumors. We found that both RP11-89K21.1 and RP11-357H14.17 were mainly located in cytosol, and it was possible that RP11-89K21.1 and RP11-357H14.17 achieved their biological functions through the ceRNA mechanism. Thus, the binding miRNAs of RP11-89K21.1 and RP11-357H14.17 were predicted by AnnoLnc. The expressions of potential miRNAs were further validated by starBase. The results showed that $22 \mathrm{miR}$ NAs families and 10 miRNAs families were associated with RP11-89K21.1 and RP11-357H14.17, respectively. There were 5 miRNAs families overlapped in the lncRNAs binding miRNAs (Fig. 5a, Table 2 and Additional file 1: Table S2), of which the expression of miR-27b, 


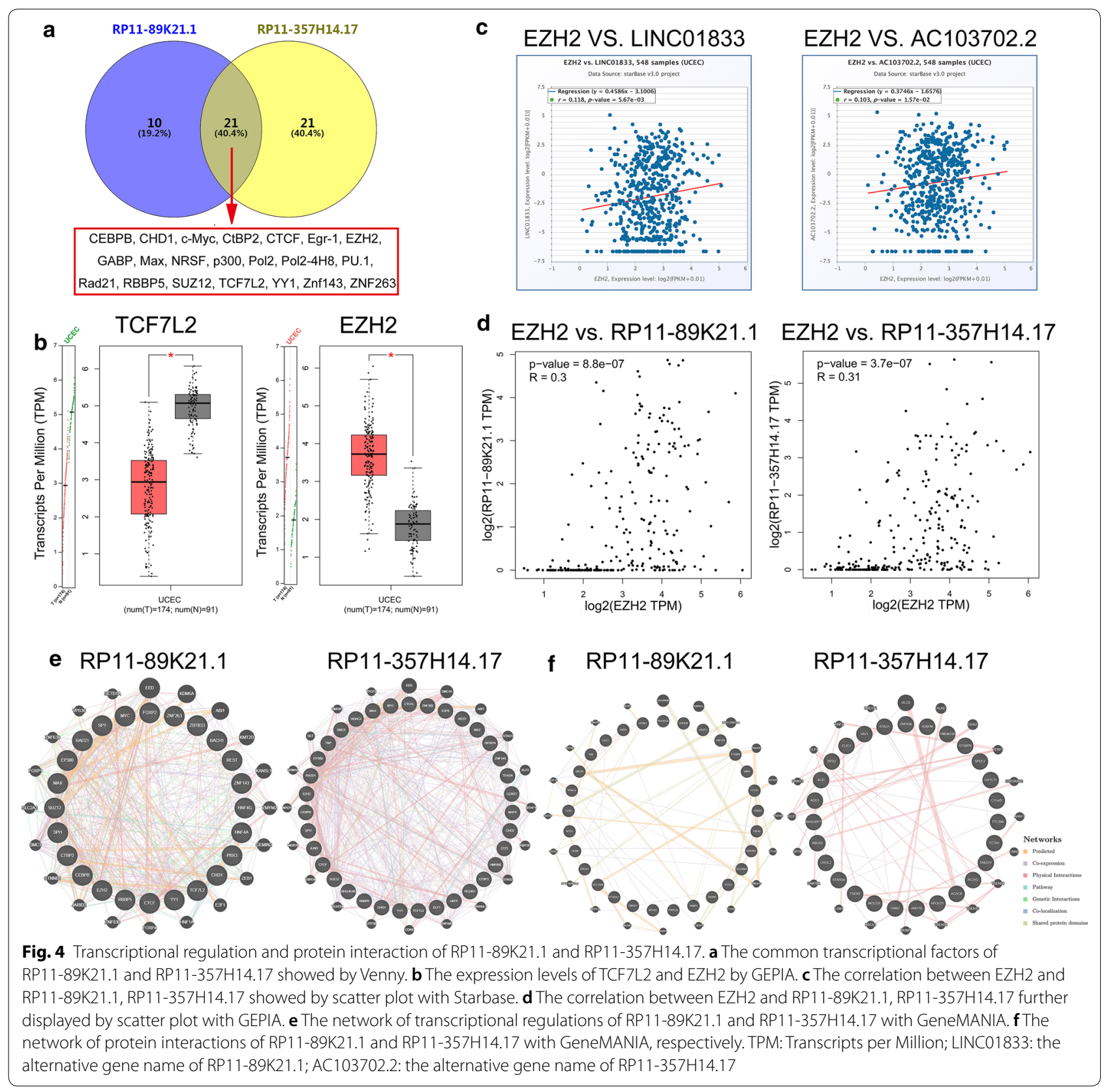

miR-4770, miR-143, miR-204 in UCEC was significantly decreased (all $P<0.05$ ) (Fig. 5b-h). The expression of miR-125a-5p, miR-125b-5p, miR-139-5p, miR-670-3p, miR-24-1-5p, miR-503 in UCEC was also significantly decreased (all $P<0.05$ ) (Additional file 1: Figure $S 2$, Table S2). For RP11-89K21.1, miR-27b, miR-4770, miR143, miR-204, miR-125a-5p, miR-125b-5p, miR-139-5p and miR-670-3p were regarded as candidate miRNAs. For RP11-357H14.17, miR-27b, miR-4770, miR-143, miR204, miR-24-1-5p and miR-503 were considered as candidate miRNAs. The targeted mRNAs of the candidate
miRNAs were further predicted by miRTarBase. As shown in Fig. 6, lncRNA-miRNA-mRNA regulatory network was constructed by Cytoscape, there were 2 lncRNAs (RP11-89K21.1 and RP11-357H14.17), 11 miRNAs and 183 target genes included in the interaction network (Fig. 6).

\section{Functional enrichment analysis of IncRNA-related targets}

To explore the potential functions and mechanisms of RP11-89K21.1 and RP11-357H14.17 in the development of UCEC, GO and KEGG enrichment analysis of 

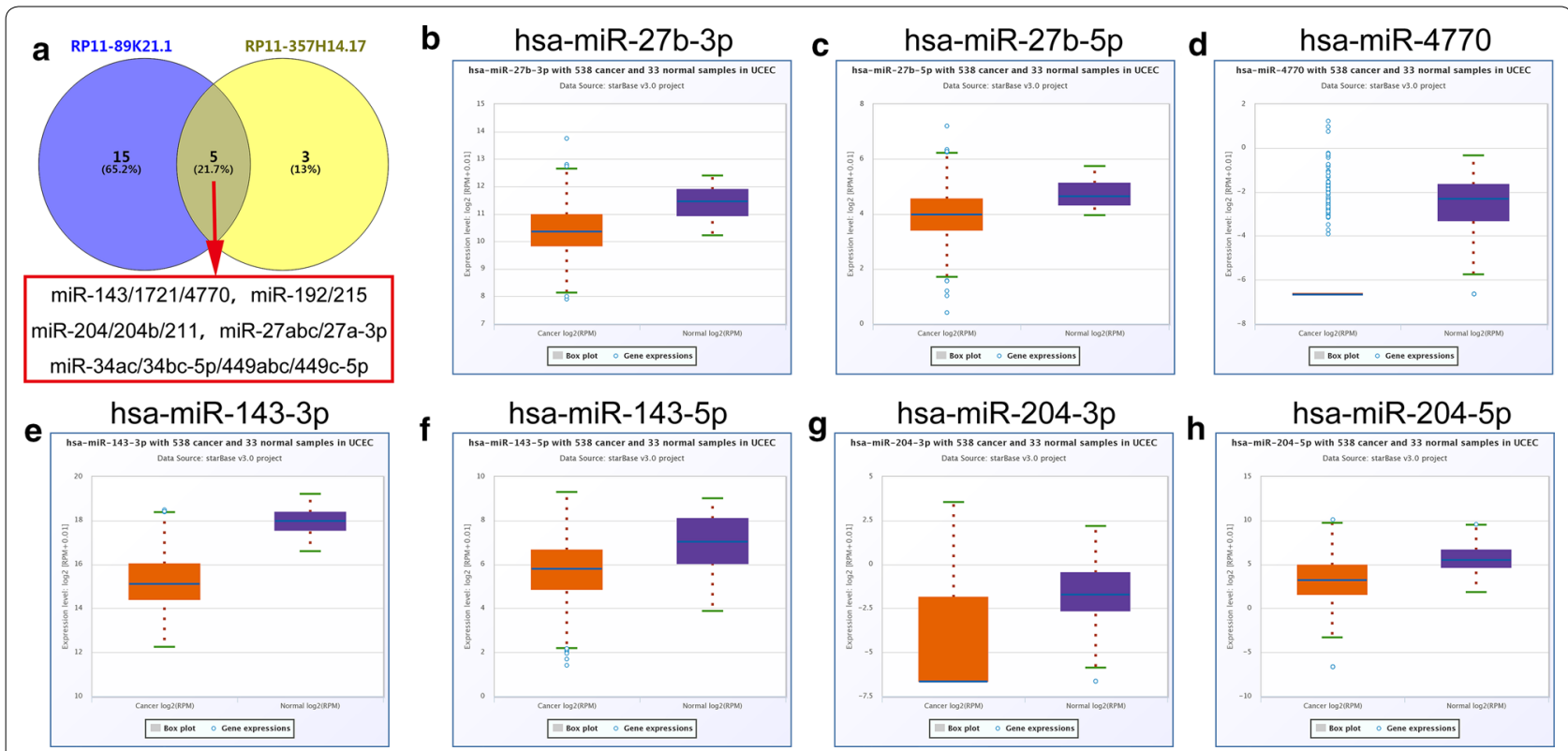

Fig. 5 The expression of common binding miRNAs of RP11-89K21.1 and RP11-357H14.17 in UCEC with starBase. a The common binding miRNAs of RP11-89K21.1 and RP11-357H14.17 showed by Venny. b-h the expression levels of miR-27b (b, c), miR-4770 (d), miR-143 (e, f), miR-204 (g, h) were downregulated in UCEC compared with normal tissues $(P<0.05)$

RP11-89K21.1 and RP11-357H14.17 targeted genes were analyzed by Metascape. GO showed that RP11-89K21.1 targeted genes primarily participated in transcription factor complex and perinuclear region of cytoplasm, also regulated proximal promoter sequence-specific DNA binding, phosphotransferase activity, kinase and growth factor binding (Fig. 7a-d, Additional file 1: Table S3-4). The biological processes of RP11-89K21.1 targeted genes mainly involved in blood vessel development, regulation of cell death and differentiation, tissue morphogenesis and response to growth factor (Fig. 7e-f and Table 3). RP11-357H14.17 targeted genes were mainly located in perinuclear region of cytoplasm, participated in cyclindependent protein kinase holoenzyme complex, adherens junction and transcription factor complex, also regulated protein kinase activity.

transcription factor and kinase binding (Fig. 8a-d, Additional file 1: Table S5, 6). RP11-89K21.1 targeted genes mainly involved in biological processes such as blood vessel development, regulation of transferase activity, apoptotic signaling pathway, regulation of cell death and cell differentiation, response to oxygen levels (Fig. 8e-f and Table 4).

KEGG enrichment analysis showed that RP1189K21.1 targeted genes were significantly enriched in pathways in cancer, endocrine resistance and microRNAs in cancer, regulated apelin signaling pathway, Th17 cell differentiation and hippo signaling pathway (Fig. 7g, h, Table 5). RP11-357H14.17 targeted genes were significantly enriched in pathways in cancer, microRNAs in cancer, PI3K-AKT signaling pathway, AGERAGE signaling pathway in diabetic complications, cell cycle and cytokine-mediated signaling pathway (Fig. 8g, h, Table 6). These signaling pathways played key roles in the occurrence and development of a variety of tumors, including endometrial carcinoma. Moreover, in order to better understand the relationship between RP1189K21.1, RP11-357H14.17 and UCEC, we performed protein-protein interaction (PPI) enrichment analysis using Metascape (Figs. 7i, 8i). The most important 10 and 8 MCODE components in PPI network, pathway and enrichment process analysis were applied to each MCODE component independently.

\section{Correlation between the expression of RP11-89K21.1,} RP11-357H14.17 and immune infiltration with ImmucLnc In order to investigate the relationship between RP1189K21.1, RP11-357H14.17 and immune infiltration, ImmucLnc database was employed to detect RP1189K21.1, RP11-357H14.17 correlated immune cell types (CD8_T cell, Macrophage, Dendritic, BMSCs and CD4_T cell, and Neutrophil). We found that the expression of RP11-89K21.1 was negatively correlated with CD8_T cell (Rs Value $=-0.159, P=0$ ) and Macrophage (Rs Value $=-0.11, P=0.01$ ), and positively correlated with CD4_T cell (Rs Value $=0.106, P=0.013)$ and Neutrophil (Rs Value $=0.138, P=0.001)$ (Table 7). The expression of RP11-357H14.17 was negatively correlated with 
Table 2 MiRNAs correlated with RP11-89K21.1 and RP11357H14.17 predicted with AnnoLnc

\begin{tabular}{|c|c|}
\hline LncRNA & MiRNA families \\
\hline \multirow[t]{22}{*}{ RP11-89K21.1 } & miR-122/122a/1352 \\
\hline & miR-125a-5p/125b-5p/351/670/4319 \\
\hline & miR-125a-5p/125b-5p/351/670/4319 \\
\hline & miR-128/128ab \\
\hline & miR-139-5p \\
\hline & miR-143/1721/4770 \\
\hline & miR-146ac/146b-5p \\
\hline & miR-146ac/146b-5p \\
\hline & miR-150/5127 \\
\hline & miR-192/215 \\
\hline & miR-194 \\
\hline & miR-1ab/206/613 \\
\hline & miR-200bc/429/548a \\
\hline & miR-204/204b/211 \\
\hline & miR-21/590-5p \\
\hline & $\mathrm{miR}-216 b / 216 b-5 p$ \\
\hline & miR-218/218a \\
\hline & miR-27abc/27a-3p \\
\hline & miR-34ac/34bc-5p/449abc/449c-5p \\
\hline & miR-455-5p \\
\hline & miR-490-3p \\
\hline & miR-7/7ab \\
\hline \multirow[t]{10}{*}{ RP11-357H14.17 } & miR-204/204b/211 \\
\hline & $\operatorname{miR}-27 a b c / 27 a-3 p$ \\
\hline & miR-192/215 \\
\hline & miR-138/138ab \\
\hline & miR-34ac/34bc-5p/449abc/449c-5p \\
\hline & miR-34ac/34bc-5p/449abc/449c-5p \\
\hline & miR-143/1721/4770 \\
\hline & miR-24/24ab/24-3p \\
\hline & miR-503 \\
\hline & miR-138/138ab \\
\hline
\end{tabular}

CD8_T cell (Rs Value $=-0.114, P=0.008)$ and positively correlated with CD4_T cell (Rs Value $=0.102, P=0.017$ ) (Table 8).

\section{Discussion}

A series of biological processes are involved in the occurrence and progression of $\mathrm{EC}$, such as abnormal expression of genes and transcription factors, dysregulation of cellular signal transduction pathway and imbalance of cell microenvironment homeostasis. Pathological changes and molecular characteristics determine the level of risk and prognosis of patients with EC. In recent years, 1ncRNAs have been identified to exert various malignant biological behaviors in EC including differentiation, proliferation, invasion and metastasis [30]. Therefore, it is valuable and helpful to explore the potential functions and molecular mechanisms of lncRNAs in EC, which contribute to prognostic prediction and therapeutic target of endometrial carcinoma.

In this study, 121 differentially expressed lncRNAs in UCEC were identified by circlncRNAnet, including 77 upregulated and 44 downregulated lncRNAs. We further confirmed for the first time that only high expressions of RP11-89K21.1 and RP11-357H14.17 were significantly associated with shortened OS and poor prognosis of patients with UCEC, which suggested that RP11-89K21.1 and RP11-357H14.17 played oncogene roles in the occurrence, progression of endometrial carcinoma. It was reported that the expression of IncRNAs were regulated by transcription factors [31]. We found that EZH2 was the common transcriptional regulator of RP11-89K21.1 and RP11-357H14.17 in endometrial carcinoma with AnnoLnc, Moreover, EZH2 was positively correlated with the expression of RP11-89K21.1 and RP11-357H14.17. Some studies have showed that lncRNA DLEU2 interacted with EZH2 to promote the proliferation, migration and invasion of hepatocellular carcinoma, thus accelerating the malignant progression of hepatocellular carcinoma [32]. In gastric cancer, IncRNA UCA1 enhanced the translation of cyclin D1 via recruiting EZH2 and further precipitated the proliferation and cell cycle progression of gastric cancer [33]. In lung cancer, the expression of lncRNA-SVUGP2 could be suppressed by EZH2 and further promoted the occurrence and development of lung cancer via $\mathrm{Wnt} / \beta$-catenin pathway [34]. These studies suggest that there exists potential regulatory mechanism between EZH2 and RP11-89K21.1, RP11$357 \mathrm{H} 14.17$ involved in the occurrence and development of endometrial carcinoma, and the specific mechanism remains to be further explored and verified.

Studies have shown that lncRNAs are located in different subcellular structures, including cytoplasm, nucleus, ribosome, cytosol and exosome. Functions and regulatory mechanisms of lncRNAs are closely associated with subcellular localization. We detected that RP1189K21.1 and RP11-357H14.17 were mainly located in cytosol. Growing evidence suggested that, in the cytoplasm and cytosol, lncRNAs not only regulated the stability and translation of mRNA, but also had an impact on the post-transcriptional modification of proteins and cell signal transduction. Based on the ceRNA hypothesis, lncRNA can competitively bind to miRNAs acting as sponge of miRNAs, detaining or adsorbing miRNAs, thus relieving the inhibition of miRNAs on downstream target genes [35, 36]. Some studies have shown that exosomal lncRNA ARSR could competitively bind to miR-34 and miR-449 to regulate the expression of AXL, c-MET, 


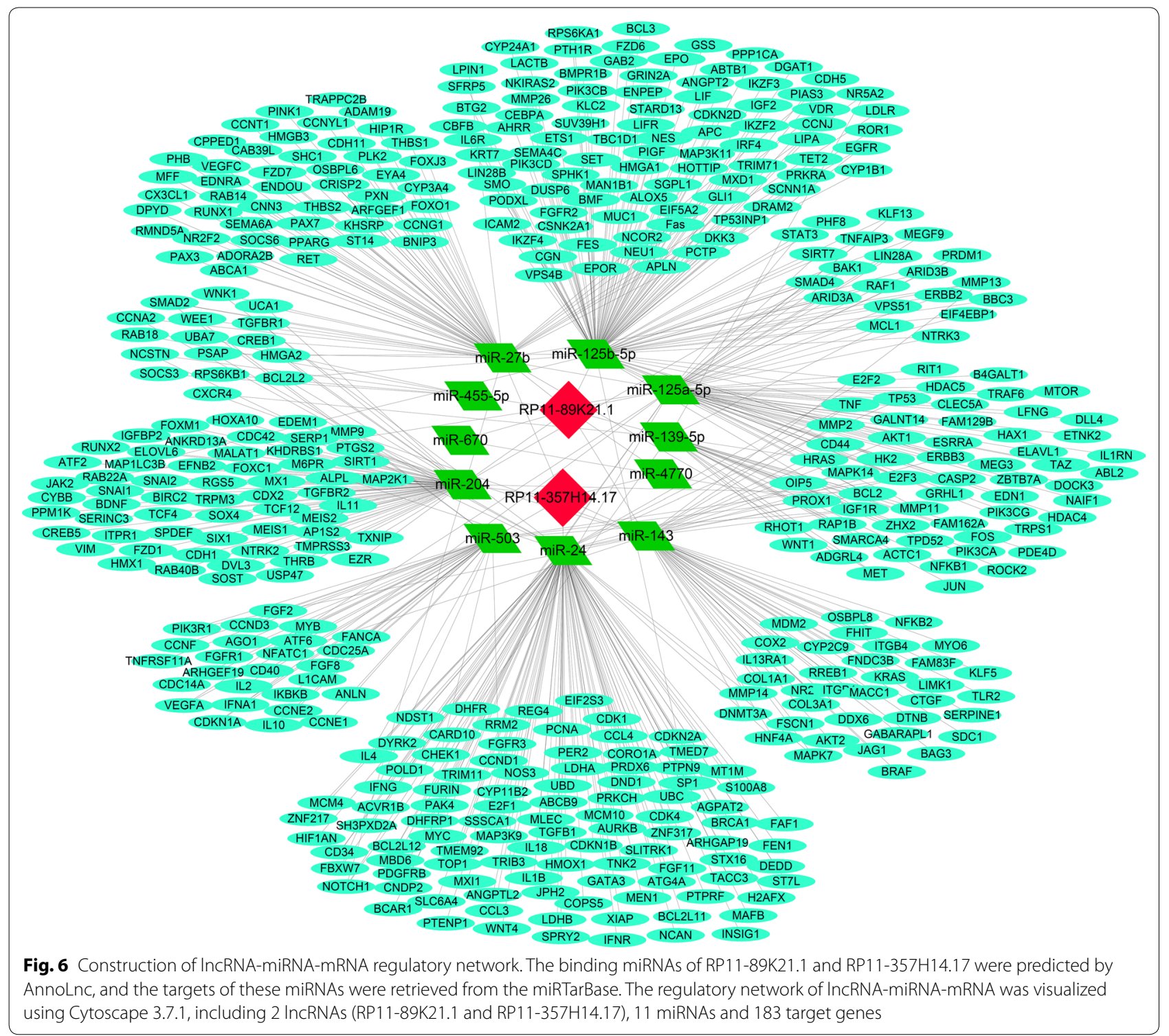

\section{(See figure on next page.)}

Fig. 7 Significant enrichment analysis of GO and KEGG pathways of target genes correlated with RP11-89K21.1 in UCEC with Metascape. a-f Cellular component $(\mathbf{a}, \mathbf{b})$, molecular function $(\mathbf{c}, \mathbf{d})$ and biological process $(\mathbf{e}, \mathbf{f})$ enrichment analysis of RP11-89K21.1-related targets colored by $P$-value with bar graph and network (Top 20). $\mathbf{g}, \mathbf{h}$ KEGG pathways enrichment analysis of RP11-89K21.1-related targets colored by $P$-value with bar graph and network (Top 20). $\mathbf{i}$ The PPI network formed by protein-protein interaction (PPI) network and the ten most significant MCODE components of RP1 1-89K21.1 target genes. GO Gene Ontology, KEGG Kyoto Encyclopedia of Genes and Genomes, MCODE Molecular Complex Detection. Above results were colored by $P$-value, where terms containing more genes tend to have a more significant $P$-value

and then promoted the drug resistance of renal cell carcinoma [37]. In prostate cancer, lncRNA TTTY15 acted as a ceRNA and negatively regulated miR-let-7 to promote expression of the target genes (CDK6, FN1) [38]. In recent years, mounting studies have shown that lncRNA, acting as ceRNA, regulated the expression of downstream oncogenes and tumor suppressor genes in endometrial carcinoma through a miRNA regulatory mechanism. In endometrial carcinoma, IncRNA HOTAIR facilitated the expression of NPM1 by negatively regulating miR-646, and thereby promoting the proliferation, migration and invasion of EC cells [39]. Maziveyi et al. 


\section{a Cellular Component}

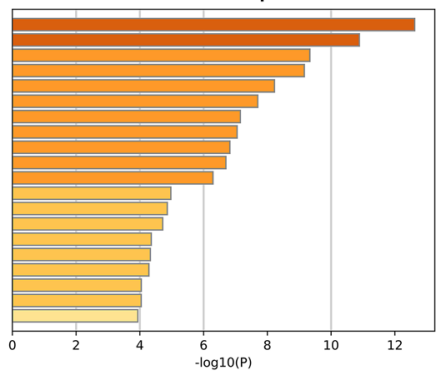

c

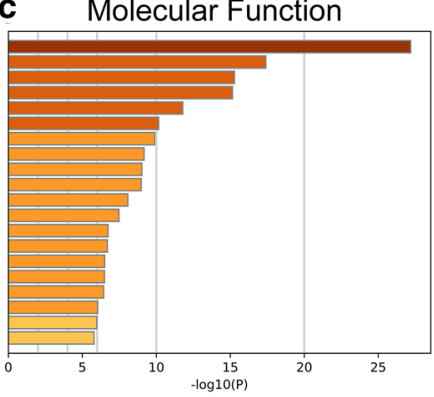

e Biological Process

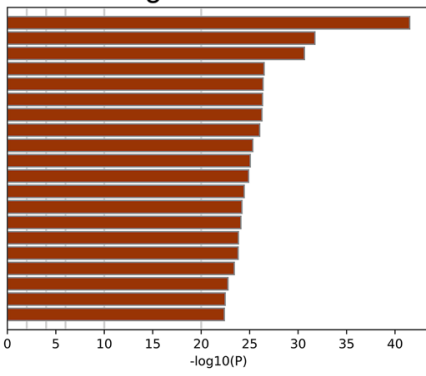

\section{g KEGG Pathway}

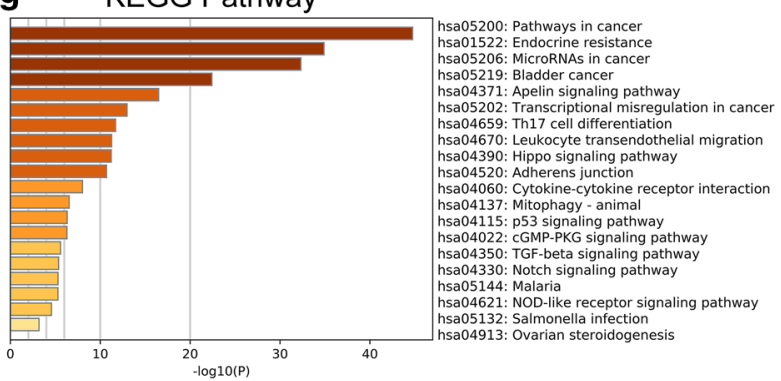

G0:0019900: kinase binding

GO:0019904: protein domain specific binding

.0003712: transcription coregulator activity

GO:0042803: protein homodimerization activity

GO:0005126: cytokine receptor binding

GO:0019207: kinase regulator activity

GO:00031625: ubiquitin protein ligase binding

G:0050839: cell adhesion molecule bin

GO:0002020: protease binding

G0:0003707: steroid hormone rement binding

G0:0001568: blood vessel development

G0:00917190: apoptotic signaling pathway

0019221: cytokinemediated signaling pathway

60:0070482: response to oxygen levels

(0.003085: epithelial cellation of kinase activity

60:0008285: negative regulation of cell proliferation

G0:1901652: response to peptide

$0: 0080135$ : regulation of cellular response to stres

60:0070997: neuron death

60:0007568: aging b 60:0005667: transcription factor complex

60:0005741: mitochondrial outer membrane

G0:0043235: receptor complex

(a)

60:0005942: phosphatidylinositol 3-kinase complex

G0:0000792: heterochromatin

60:0045121: membrane raft

G0:0005635: nuclear envelope

GO:0017053: transcriptional repressor complex

GO:0098978: glutamatergic synapse

G0:0097136: Bcl-2 family protein complex

protein complex
kappaB complex

GO:0098552: side of membrane
0:0035985: senescence-associated heterochromatin focus

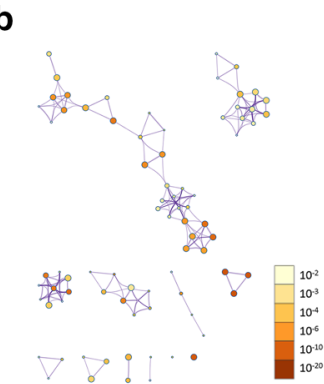

d

GO:0000987: proximal promoter sequence-specific DNA binding

GO:0052812: phosphatidylinositol-3,4-bisphosphate 5-kinase activity

G0:0016538: cyclin-dependent protein serine/threonine kinase regulator activity

GO:0001223: transcription coactivator binding

f

GO:0002521: leukocyte differentiation

c.0008729: tissue mophogenesis

G0:0040008: regulation of growth

60:0048555: response to steroid hormone

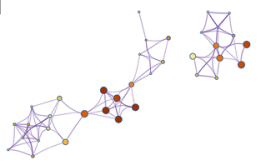

$\therefore *$ \%
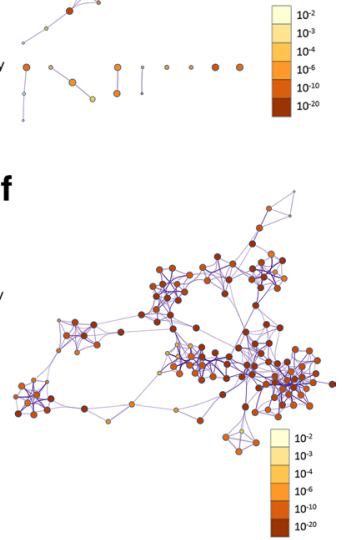

h
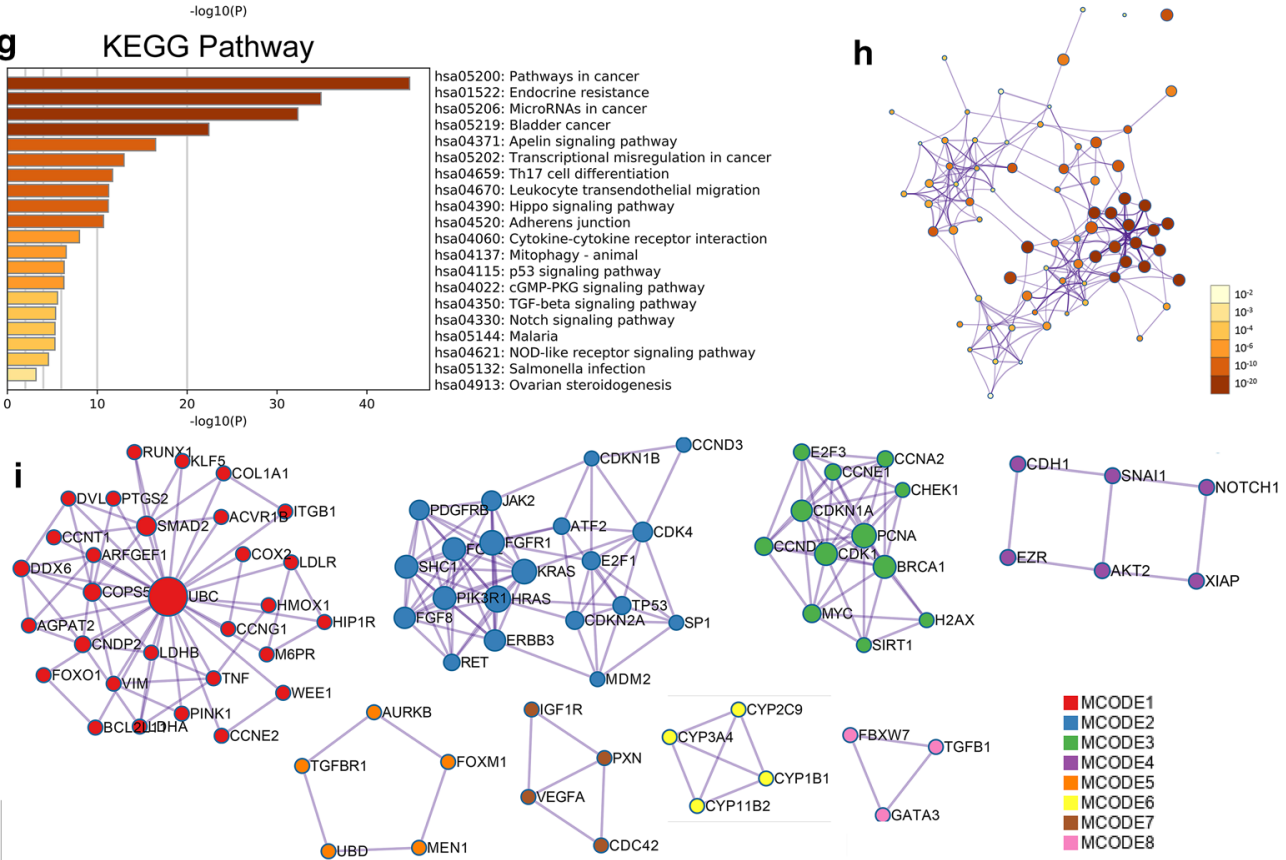

$\underbrace{\text { OfBXw7 }}_{\text {OGATA3 }}$

MCODE1

MCODE2

MCODE 3
MCODE4

MCODE4

MCODE5
MCODE6

MCODE6
MCODE7

MCODE8 
Table 3 Significantly enriched GO annotations (Biological Processes) of RP11-89K21.1 in endometrial carcinoma with Metascape (Top 20)

\begin{tabular}{|c|c|c|c|c|c|c|}
\hline GO & Category & Description & Count & $\%$ & $\log 10(P)$ & $\log 10(q)$ \\
\hline GO:0001568 & GO Biological Processes & Blood vessel development & 76 & 9.78 & -41.51 & -37.31 \\
\hline GO:0097190 & GO Biological Processes & Apoptotic signaling pathway & 59 & 9.72 & -31.73 & -28.00 \\
\hline GO:0010942 & GO Biological Processes & Positive regulation of cell death & 63 & 8.53 & -30.65 & -27.15 \\
\hline GO:0002521 & GO Biological Processes & Leukocyte differentiation & 50 & 9.58 & -26.49 & -23.19 \\
\hline GO:0048729 & GO Biological Processes & Tissue morphogenesis & 56 & 8.24 & -26.38 & -23.18 \\
\hline GO:0007169 & GO Biological Processes & $\begin{array}{l}\text { Transmembrane receptor protein tyrosine } \\
\text { kinase signaling pathway }\end{array}$ & 58 & 7.87 & -26.32 & -23.17 \\
\hline GO:0019221 & GO Biological Processes & Cytokine-mediated signaling pathway & 60 & 7.54 & -26.26 & -23.14 \\
\hline GO:0070482 & GO Biological Processes & Response to oxygen levels & 44 & 11.17 & -26.03 & -22.98 \\
\hline GO:0040008 & GO Biological Processes & Regulation of growth & 55 & 8.02 & -25.32 & -22.42 \\
\hline GO:0033674 & GO Biological Processes & Positive regulation of kinase activity & 52 & 8.48 & -25.06 & -22.18 \\
\hline GO:0030855 & GO Biological Processes & Epithelial cell differentiation & 58 & 7.38 & -24.89 & -22.03 \\
\hline GO:0008285 & GO Biological Processes & Negative regulation of cell proliferation & 57 & 7.37 & -24.43 & -21.59 \\
\hline GO:1901652 & GO Biological Processes & Response to peptide & 48 & 8.99 & -24.20 & -21.38 \\
\hline GO:0007507 & GO Biological Processes & Heart development & 50 & 8.49 & -24.09 & -21.29 \\
\hline GO:0080135 & GO Biological Processes & Regulation of cellular response to stress & 56 & 7.32 & -23.84 & -21.07 \\
\hline GO:0070997 & GO Biological Processes & Neuron death & 40 & 11.27 & -23.81 & -21.05 \\
\hline GO:0048545 & GO Biological Processes & Response to steroid hormone & 41 & 10.62 & -23.41 & -20.67 \\
\hline GO:0030155 & GO Biological Processes & Regulation of cell adhesion & 53 & 7.40 & -22.76 & -20.09 \\
\hline GO:0007568 & GO Biological Processes & Aging & 37 & 11.60 & -22.47 & -19.84 \\
\hline GO:0045596 & GO Biological Processes & Negative regulation of cell differentiation & 54 & 7.11 & -22.38 & -19.76 \\
\hline
\end{tabular}

(See figure on next page.)

Fig. 8 Significant enrichment analysis of GO and KEGG pathways of target genes correlated with RP1 1-357H14.17 in UCEC with Metascape. a-f Cellular component $(\mathbf{a}, \mathbf{b})$, molecular function $(\mathbf{c}, \mathbf{d})$ and biological process $(\mathbf{e}, \mathbf{f})$ enrichment analysis of RP11-357H14.17-ralated targets colored by $P$-value with bar graph and network (Top 20). $\mathbf{g}$, h KEGG pathways enrichment analysis of RP11-357H14.17-related targets colored by $P$-value with bar graph and network (Top 20). $\mathbf{i}$ The PPI network formed by protein-protein interaction (PPI) network and the ten most significant MCODE components of RP11-357H14.17 target genes. GO: Gene Ontology; KEGG: Kyoto Encyclopedia of Genes and Genomes; MCODE: Molecular Complex Detection. Above results were colored by $P$-value, where terms containing more genes tend to have a more significant $P$-value

reported that lncRNA TUSC7 promoted the expression of SOCS4 (SOCS5) through acing as sponge of miR-616, thus inhibiting the proliferation, migration and invasion of endometrial carcinoma [40]. We further explored the potential role of ceRNA network mechanism regulated by RP11-89K21.1 and RP11-357H14.17 in the progression of EC. The binding miRNAs of RP11-89K21.1 and RP11-357H14.17 were retrieved from miRTarBase and 4 overlapped miRNAs (miR-27b, miR-4770, miR-143, miR-204) were downregulated in UCEC, and other RP1189K21.1 binding miRNA (miR-125a-5p, miR-125b-5p, miR-139-5p, miR-670-3p) and RP11-357H14.17 binding miRNA (miR-24-1-5p, miR-503) were also decreased in UCEC. miRNAs were involved in a variety of malignant biological behaviors and mechanisms such as proliferation, invasion and migration of tumors. The expression of miR-27b-3, miR-204-5p was decreased in EC, and high expression of miR-27b-3 and miR-204-5p could significantly inhibit the proliferation, migration and invasion of EC cells $[41,42]$. The expression of miR-143 and miR-503 were also downregulated in EC, and high expression of miR-503 inhibited the proliferation and cell cycle of EC cells through negatively regulating CCND1 $[43,44]$. These findings indicated that RP11-89K21.1 and RP11-357H14.17 may play oncogene roles in endometrial carcinoma by regulating candidate miRNAs and their targeted genes. Therefore, we have successfully constructed a new IncRNA-miRNA-mRNA ceRNA regulatory network associated with the prognosis of patients with EC, and further experiments are required to verify molecular mechanisms in the regulatory network.

Many studies have shown that lncRNAs affects the initiation and development of tumors by regulating a variety of molecular mechanisms and signaling pathways. Researchers observed lncRNA LSINCT5 promoted proliferation, invasion and metastasis of EC cells 


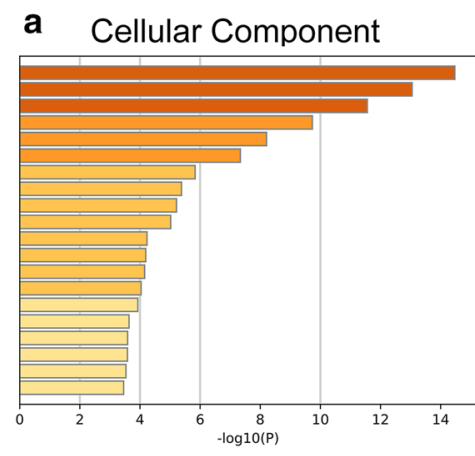

C

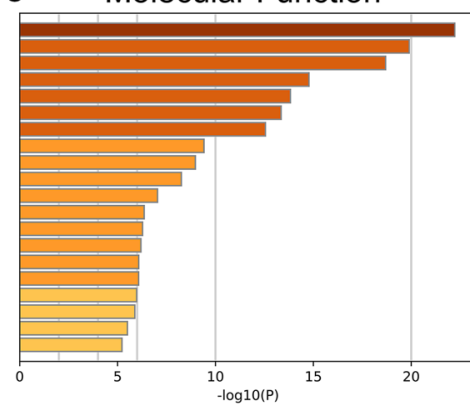

e

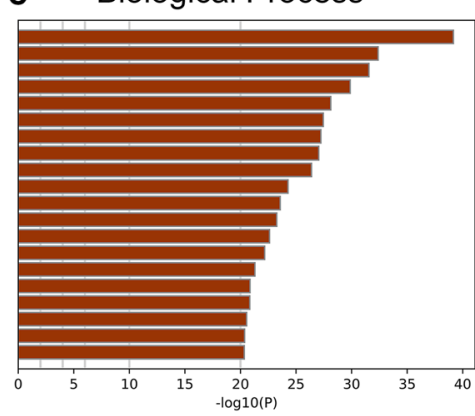

g KEGG Pathway

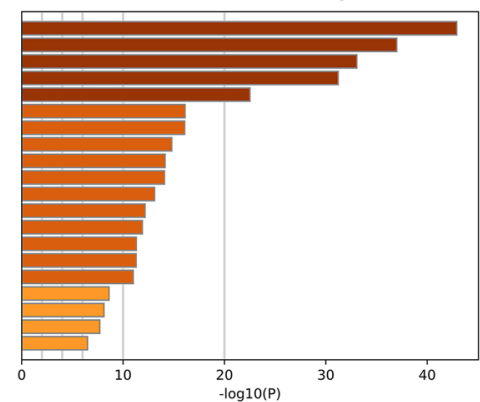

b

GO:0048471: perinuclear region of cytoplasm

07: cyclin-dependent protein kinase holoenzyme complex GO:0005912: adherens junction

GO:0045121: membrane raft

GO:0031252: cell leading edge

GO:0031012: extracellular matrix

60:0005635: nuclear envelope

GO:0000792: heterochromatin

GO:0071141: SMAD protein complex

GO:0005741: mitochondrial outer membran

GO:0016235: aggresome

GO:0034399: nuclear periphery

GO:0005657: replication fork

GO:0005769: early endosome

GO:0031256: leading edge membrane

(a)

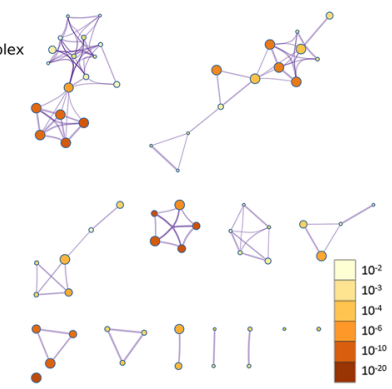

GO:0004672: protein kinase activity GO:0019900: kinase binding GO: 0019904: protein domain specific GO:0031625: ubiquitin protein ligase binding GO:0005126: cytokine receptor binding GO:0002020: protease binding GO:0050839: cell adhesion molecule binding 60.0019602 : phosphatase binding

G0.0035497: CAMP response element binding GO:0042803: protein homodimerization activity GO:0008022: protein C-terminus binding GO:0001223: transcription coactivator binding GO:0004712: protein serine/threonine/tyrosine GO:0003707: steroid hormone receptor activity

d
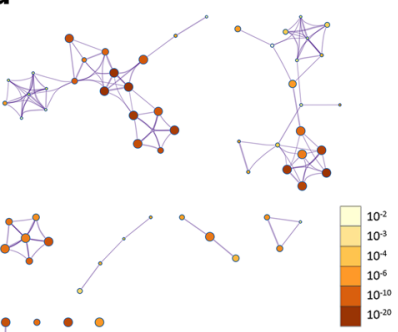
.

GO:0001568: blood vessel development GO:0001568: blood vessel development GO:0097190: apoptotic signaling pathway GO:0070482: response to oxygen levels GO:0070848: response to growth factor GO:0048732: gland development GO:0080135: regulation of cellular response to stress (0.0048729: tissue morphogenesis GO:0048729: tissue morphogenesis GO:0072593: reactive oxygen species metabolic process GO:0019221: cytokine-mediated signaling pathway Go:0048545: response to steroid hormone GO:0010035: response to inorg

GO:0097191: extrinsic apoptotic signaling pathway GO:0002521: leukocyte differentiation

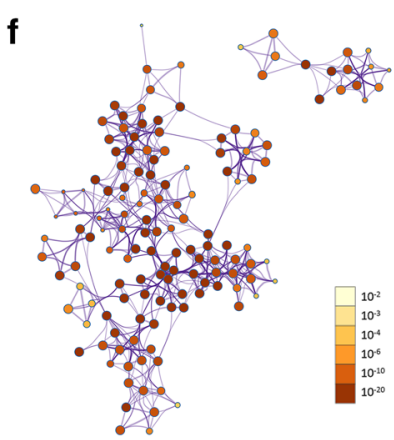

h

hsa05200: Pathways in cancer gathway hsa04933: AGE-RAGE signaling pathway in diabetic complications o hsa05202: Transcriptional misregulation in cance hsa04668: TNF signaling pathway hsa04060: Cytokine-cytokine receptor interaction ha04066: Hif-1 signaling pathway hsa05162: Measle

hsa05216: Thyroid cancer hsa04520: Adherens junction hsa04659: Th17 cell differentiation 5a049:: Hippo signaling pathway hsa04350: TGF-beta signaling pathway
hsa04670: Leukocyte transendothelial migration
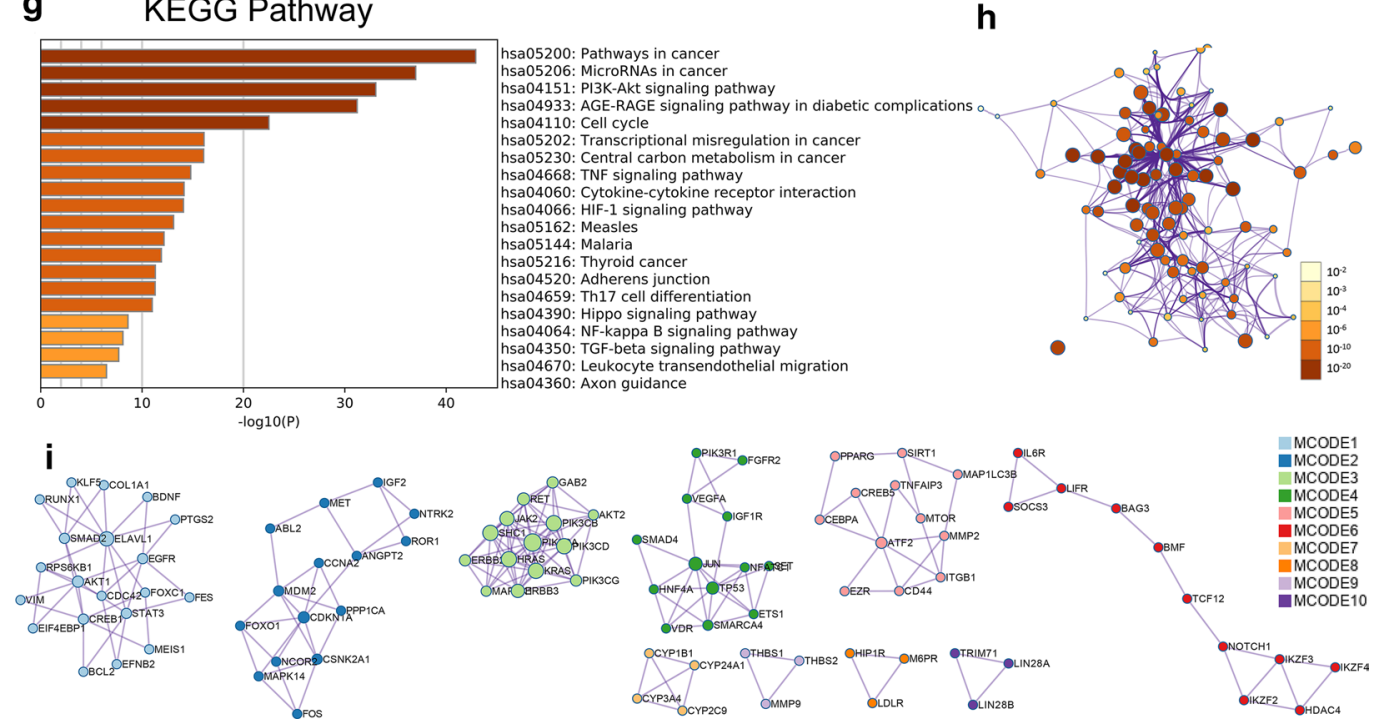
Table 4 Significantly enriched GO annotations (Biological Processes) of RP11-357H14.17 in endometrial carcinoma with Metascape (Top 20)

\begin{tabular}{|c|c|c|c|c|c|c|}
\hline GO & Category & Description & Count & $\%$ & $\log 10(P)$ & $\log 10(q)$ \\
\hline GO:0001568 & GO Biological Processes & Blood vessel development & 70 & 9.01 & -39.16 & -34.96 \\
\hline GO:0051347 & GO Biological Processes & Positive regulation of transferase activity & 60 & 8.67 & -32.38 & -28.66 \\
\hline GO:0097190 & GO Biological Processes & Apoptotic signaling pathway & 56 & 9.23 & -31.55 & -27.95 \\
\hline GO:0010942 & GO Biological Processes & Positive regulation of cell death & 59 & 7.98 & -29.87 & -26.52 \\
\hline GO:0070482 & GO Biological Processes & Response to oxygen levels & 44 & 11.17 & -28.12 & -24.97 \\
\hline GO:0045596 & GO Biological Processes & Negative regulation of cell differentiation & 57 & 7.51 & -27.45 & -24.42 \\
\hline GO:0070848 & GO Biological Processes & Response to growth factor & 56 & 7.61 & -27.24 & -24.24 \\
\hline GO:0048732 & GO Biological Processes & Gland development & 45 & 10.18 & -27.04 & -24.07 \\
\hline GO:0080135 & GO Biological Processes & Regulation of cellular response to stress & 56 & 7.32 & -26.39 & -23.49 \\
\hline GO:0040008 & GO Biological Processes & Regulation of growth & 51 & 7.43 & -24.28 & -21.47 \\
\hline GO:0048729 & GO Biological Processes & Tissue morphogenesis & 50 & 7.35 & -23.57 & -20.81 \\
\hline GO:0009611 & GO Biological Processes & Response to wounding & 50 & 7.24 & -23.26 & -20.51 \\
\hline GO:0072593 & GO Biological Processes & Reactive oxygen species metabolic process & 34 & 11.89 & -22.61 & -19.92 \\
\hline GO:0019221 & GO Biological Processes & Cytokine-mediated signaling pathway & 52 & 6.53 & -22.18 & -19.53 \\
\hline GO:0048545 & GO Biological Processes & Response to steroid hormone & 37 & 9.59 & -21.30 & -18.68 \\
\hline GO:1901652 & GO Biological Processes & Response to peptide & 42 & 7.87 & -20.86 & -18.26 \\
\hline GO:0010035 & GO Biological Processes & Response to inorganic substance & 43 & 7.62 & -20.83 & -18.25 \\
\hline GO:0070997 & GO Biological Processes & Neuron death & 35 & 9.86 & -20.55 & -18.00 \\
\hline GO:0097191 & GO Biological Processes & Extrinsic apoptotic signaling pathway & 29 & 12.95 & -20.37 & -17.82 \\
\hline GO:0002521 & GO Biological Processes & Leukocyte differentiation & 41 & 7.85 & -20.34 & -17.80 \\
\hline
\end{tabular}

Table 5 Significantly enriched KEGG pathway of RP11-89K21.1 in endometrial carcinoma with Metascape (Top 20)

\begin{tabular}{|c|c|c|c|c|c|c|}
\hline GO & Category & Description & Count & $\%$ & $\log 10(P)$ & $\log 10(q)$ \\
\hline hsa05200 & KEGG Pathway & Pathways in cancer & 61 & 15.44 & -44.75 & -42.06 \\
\hline hsa01522 & KEGG Pathway & Endocrine resistance & 32 & 33.33 & -34.89 & -32.68 \\
\hline hsa05206 & KEGG Pathway & MicroRNAs in cancer & 45 & 15.05 & -32.32 & -30.23 \\
\hline hsa05219 & KEGG Pathway & Bladder cancer & 18 & 43.90 & -22.42 & -20.98 \\
\hline hsa04371 & KEGG Pathway & Apelin signaling pathway & 22 & 15.94 & -16.49 & -15.42 \\
\hline hsa05202 & KEGG Pathway & Transcriptional misregulation in cancer & 21 & 11.67 & -12.98 & -12.02 \\
\hline hsa04659 & KEGG Pathway & Th17 cell differentiation & 16 & 14.95 & -11.70 & -10.80 \\
\hline hsa04670 & KEGG Pathway & Leukocyte transendothelial migration & 16 & 14.04 & -11.27 & -10.39 \\
\hline hsa04390 & KEGG Pathway & Hippo signaling pathway & 18 & 11.69 & -11.22 & -10.36 \\
\hline hsa04520 & KEGG Pathway & Adherens junction & 13 & 18.06 & -10.69 & -9.84 \\
\hline hsa04060 & KEGG Pathway & Cytokine-cytokine receptor interaction & 19 & 7.04 & -8.02 & -7.26 \\
\hline hsa04137 & KEGG Pathway & Mitophagy-animal & 9 & 13.85 & -6.54 & -5.82 \\
\hline hsa04115 & KEGG Pathway & p53 signaling pathway & 9 & 13.04 & -6.31 & -5.60 \\
\hline hsa04022 & KEGG Pathway & cGMP-PKG signaling pathway & 13 & 7.98 & -6.28 & -5.58 \\
\hline hsa04350 & KEGG Pathway & TGF-beta signaling pathway & 9 & 10.71 & -5.58 & -4.90 \\
\hline hsa04330 & KEGG Pathway & Notch signaling pathway & 7 & 14.58 & -5.36 & -4.69 \\
\hline hsa05144 & KEGG Pathway & Malaria & 7 & 14.29 & -5.30 & -4.64 \\
\hline hsa04621 & KEGG Pathway & NOD-like receptor signaling pathway & 12 & 7.06 & -5.29 & -4.64 \\
\hline hsa05132 & KEGG Pathway & Salmonella infection & 8 & 9.30 & -4.56 & -3.94 \\
\hline hsa04913 & KEGG Pathway & Ovarian steroidogenesis & 5 & 10.00 & -3.19 & -2.61 \\
\hline
\end{tabular}


Table 6 Significantly enriched KEGG pathway of RP11-357H14.17 in endometrial carcinoma with Metascape (Top 20)

\begin{tabular}{|c|c|c|c|c|c|c|}
\hline GO & Category & Description & Count & $\%$ & $\log 10(P)$ & $\log 10(q)$ \\
\hline hsa05200 & KEGG Pathway & Pathways in cancer & $57 / 395$ & 14.43 & -42.91 & -40.22 \\
\hline hsa05206 & KEGG Pathway & MicroRNAs in cancer & $47 / 299$ & 15.72 & -36.99 & -34.60 \\
\hline hsa04151 & KEGG Pathway & PI3K-Akt signaling pathway & $46 / 342$ & 13.45 & -33.06 & -30.84 \\
\hline hsa04933 & KEGG Pathway & $\begin{array}{l}\text { AGE-RAGE signaling pathway in diabetic } \\
\text { complications }\end{array}$ & 29/99 & 29.29 & -31.22 & -29.22 \\
\hline hsa04110 & KEGG Pathway & Cell cycle & $25 / 124$ & 20.16 & -22.51 & -21.02 \\
\hline hsa05202 & KEGG Pathway & Transcriptional misregulation in cancer & $23 / 180$ & 12.78 & -16.11 & -14.80 \\
\hline hsa05230 & KEGG Pathway & Central carbon metabolism in cancer & $16 / 65$ & 24.62 & -16.07 & -14.78 \\
\hline hsa04668 & KEGG Pathway & TNF signaling pathway & $18 / 108$ & 16.67 & -14.80 & -13.55 \\
\hline hsa04060 & KEGG Pathway & Cytokine-cytokine receptor interaction & $25 / 270$ & 9.26 & -14.14 & -12.92 \\
\hline hsa04066 & KEGG Pathway & HIF-1 signaling pathway & $17 / 101$ & 16.83 & -14.08 & -12.88 \\
\hline hsa05162 & KEGG Pathway & Measles & $18 / 134$ & 13.43 & -13.10 & -11.98 \\
\hline hsa05144 & KEGG Pathway & Malaria & $12 / 49$ & 24.49 & -12.16 & -11.06 \\
\hline hsa05216 & KEGG Pathway & Thyroid cancer & $10 / 29$ & 34.48 & -11.90 & -10.86 \\
\hline hsa04520 & KEGG Pathway & Adherens junction & $13 / 72$ & 18.06 & -11.31 & -10.30 \\
\hline hsa04659 & KEGG Pathway & Th17 cell differentiation & $15 / 107$ & 14.02 & -11.29 & -10.29 \\
\hline hsa04390 & KEGG Pathway & Hippo signaling pathway & $17 / 154$ & 11.04 & -11.00 & -10.02 \\
\hline hsa04064 & KEGG Pathway & NF-kappa B signaling pathway & $12 / 95$ & 12.63 & -8.61 & -7.76 \\
\hline hsa04350 & KEGG Pathway & TGF-beta signaling pathway & $11 / 84$ & 13.10 & -8.11 & -7.27 \\
\hline hsa04670 & KEGG Pathway & Leukocyte transendothelial migration & $12 / 114$ & 10.53 & -7.70 & -6.86 \\
\hline hsa04360 & KEGG Pathway & Axon guidance & $13 / 175$ & 7.43 & -6.49 & -5.73 \\
\hline
\end{tabular}

Table 7 Correlation of RP11-89K21.1 with immune infiltration analyzed by ImmucLnc

\begin{tabular}{|c|c|c|c|c|c|}
\hline Cancer & LncRNA ID & LncRNA symbol & Immune cell & $P$ value & Rs value ${ }^{\mathrm{b}}$ \\
\hline UCEC & ENSG00000259439 & LINC01833 & CD8_T cell & 0 & -0.159 \\
\hline UCEC & ENSG00000259439 & LINC01833 & Macrophage & 0.01 & -0.11 \\
\hline UCEC & ENSG00000259439 & LINC01833 & Dendritic & 0.981 & -0.001 \\
\hline UCEC & ENSG00000259439 & LINC01833 & B_cell & 0.404 & 0.036 \\
\hline UCEC & ENSG00000259439 & LINC01833 & CD4_T cell & 0.013 & 0.106 \\
\hline UCEC & ENSG00000259439 & LINC01833 & Neutrophil & 0.001 & 0.138 \\
\hline
\end{tabular}

a LINC01833 the alternative gene name of RP11-89K21.1, ${ }^{\mathrm{b}}$ The correlation coefficient

Table 8 Correlation of RP11-357H14.17 with immune infiltration analyzed by ImmucLnc

\begin{tabular}{lllllc}
\hline Cancer & LncRNA ID & LncRNA symbol & Immune cell & P value & Rs value $^{\mathbf{a}}$ \\
\hline UCEC & ENSG00000272763 & RP11-357H14.17 & CD8_T cell & 0.008 & -0.114 \\
UCEC & ENSG00000272763 & RP11-357H14.17 & Dendritic & 0.072 & -0.077 \\
UCEC & ENSG00000272763 & RP11-357H14.17 & Macrophage & 0.101 & -0.07 \\
UCEC & ENSG00000272763 & RP11-357H14.17 & B_cell & 0.279 & -0.046 \\
UCEC & ENSG00000272763 & RP11-357H14.17 & Neutrophil & 0.307 & -0.044 \\
UCEC & ENSG00000272763 & RP11-357H14.17 & CD4_T cell & 0.017 & 0.102 \\
\hline
\end{tabular}

a The correlation coefficient

by regulating HMGA2/Wnt/ $\beta$-catenin signaling pathway [45]. LncRNA OGFRP1 promoted the malignant progression of endometrial carcinoma by regulating the miR1243p/SIRT1 axis and activating the PI3K/AKT/GSK-3 $\beta$ pathway [46]. In order to better clarify the biological function, molecular mechanism and regulatory network of RP11-89K21.1 and RP11-357H14.17-related targeting genes in EC, we carried out GO and KEGG enrichment 
analysis with Metascape. It was found that RP11-89K21.1 and RP11-357H14.17-related targeting genes were mainly involved in vasculature development, tissue morphogenesis, cell growth and differentiation, regulation of protein kinase activity and cellular response to stress. KEGG enrichment analysis showed that RP11-89K21.1 and RP11-357H14.17-related targeting genes were significantly enriched in microRNAs in cancer, cytokinemediated signaling pathway, transmembrane receptor protein tyrosine kinase signaling pathway, apoptotic signaling pathway. The above signaling pathways were closely associated the progression and biological behaviors of EC [47]. Therefore, we speculated that RP11-89K21.1 and RP11-357H14.17 can affect the occurrence, development and biological behavior of EC by regulating the above tumor-related pathways, which provided more evidences for further exploring the molecular mechanisms of RP1189K21.1 and RP11-357H14.17 in endometrial carcinoma.

Accumulating studies have demonstrated that a large number of immune cells and cytokines can be observed in EC, which can enhance the endogenous anti-tumor immune response and affect prognostic value and immunotherapy of EC. Immunotherapy plays a well-established role in the treatment of EC. Our results showed that the expression of RP11-89K21.1 was negatively correlated with CD8_T cell, Macrophage and positively correlated with CD4_T cell, Neutrophil. The expression of RP11357H14.17 was negatively correlated with CD8_Tcell and positively correlated with CD4_Tcell. Studies have shown that CD8_T cell and Macrophage could participate in the malignant progression of EC and serve as a potential therapeutic target for EC [48]. CD4_T cell could promote the capacity of initiating CD4_T cell rapidly by mediating immune response, and kill tumor cells directly or indirectly by stimulating and recruiting CD8_T cell cells or other immune cells [49]. The number of CD4_T cells in peripheral blood of patients with EC was significantly increased [50]. Neutrophil was the main type of immune cells in tumors, which can eliminate pathogens and prevent host from being infected by microorganisms, playing a key role in chemotherapy resistance and anti-angiogenesis therapy of tumors [51]. Some studies have displayed that neutrophils were closely correlated with survival and prognosis of patients with EC [52]. The results showed that the above immune cells were of great value in the occurrence, development and prognosis of EC. However, it's not sufficient to conclude that RP11-89K21.1 and RP11-357H14.17 play critical role in regulating the infiltration of immune cells in tumor microenvironment because of the small correlation coefficients (Rs value), and further experiments are required to verify the function of RP11-89K21.1 and RP11-357H14.17 in immune infiltration.

\section{Conclusion}

In summary, with a series of integrated databases, we demonstrated for the first time that high expressions of RP11-89K21.1 and RP11-357H14.17 were closely associated with the poor prognosis of patients with EC. We further identified transcriptional regulatory factors, co-expressed genes, interacting proteins of RP1189K21.1 and RP11-357H14.17. Regulatory networks of biological function, signaling pathways may contribute to illuminate the potential function and mechanism of RP11-89K21.1 and RP11-357H14.17 in EC. Moreover, we speculated that the ceRNA network associated with RP11-89K21.1 and RP11-357H14.17 provided novel and valuable insights into the molecular mechanisms underlying the initiation and progression of EC. Therefore, RP11-89K21.1 and RP11-357H14.17 can potentially be identified as tumor biomarkers for early diagnosis, prognosis evaluation and therapeutic targets of EC. Although existing research may not be optimal, we think it should be adequate to make a conclusion that RP11-89K21.1 and RP11-357H14.17 contributed to poor prognosis in EC. The functional experiments of RP11-89K21.1 and RP11-357H14.17 will be further conducted in the follow-up study.

\section{Supplementary information}

Supplementary information accompanies this paper at https://doi. org/10.1186/s12935-020-01359-9.

Additional file 1. The expression, prognosis, predicting miRNAs and functional analysis of RP11-89K21.1 and RP11-357H14.17

Additional file 2. Co-expressed genes correlated with RP11-89K21.1

Additional file 3. Co-expressed genes correlated with RP11-357H14.17

Additional file 4. The binding proteins associated with RP11-89K21.1

Additional file 5. The transcription factors correlated with RP11-89K21.1 Additional file 6. The binding proteins associated with RP11-357H14.17

Additional file 7. The transcription factors correlated with RP11-357H14.17

\section{Abbreviations}

EC: Endometrial carcinoma; IncRNAs: Long noncoding RNAs; UCEC: The Uterine Corpus Endometrial Carcinoma; TCGA: The Cancer Genome Atlas; ceRNA: Competing endogenous RNA; OS: Overall survival; TF: Transcriptional factor; PPI: Protein-protein interaction; GO: Gene Ontology; KEGG: Kyoto Encyclopedia of Genes and Genomes; MCODE: Molecular Complex Detection; EZH2: Enhancer of zeste 2 polycomb repressive complex 2 subunit; TPM:Transcripts per Million.

\section{Acknowledgements}

Not applicable.

\section{Authors' contributions}

$L G, B L$ designed and analyzed the study. $L G$ and $X N$ drafted the manuscript. $W Z, R G, Y Q$ participated in data acquisition and analysis. YH, XL contributed to the data interpretation $\mathrm{QL}$ and $J$ contributed to critical review and manuscript revision. All authors participated in manuscript editing. All authors read and approved the final manuscript. 


\section{Funding}

This work was supported by grants from the National Natural Science Foundation of China (No. 81672590 and No. 81472437) and Shengjing Freedom researchers' plan (No.201804). The funding body had no role in the design or conduct of the study.

\section{Availability of data and materials}

The datasets used or analyzed during the current study are available from the corresponding author upon reasonable request.

\section{Ethics approval and consent to participate}

No ethical approval nor informed consent was required in this study due to the public-availability of the data used.

\section{Consent for publication}

All authors consent to publication.

\section{Competing interests}

The authors declare that there are no conflicts of interest.

\begin{abstract}
Author details
${ }^{1}$ Department of Obstetrics and Gynaecology, Shengjing Hospital of China Medical University, No.36 Sanhao Street, Heping District, Shenyang 110004 Liaoning, China. ${ }^{2}$ Key Laboratory of Maternal-Fetal Medicine of Liaoning Province, Key Laboratory of Obstetrics and Gynecology of Higher Education of Liaoning Province, Liaoning, China.
\end{abstract}

Received: 15 April 2020 Accepted: 16 June 2020

Published online: 24 June 2020

\section{References}

1. Siegel RL, Miller KD, Jemal A. Cancer statistics, 2019. CA Cancer J Clin. 2019;69(1):7-34. https://doi.org/10.3322/caac.21551.

2. Arend RC, Jones BA, Martinez A, Goodfellow P. Endometrial cancer: molecular markers and management of advanced stage disease. Gynecol Oncol. 2018;150(3):569-80. https://doi.org/10.1016/j.ygyno.2018.05.015.

3. Okazaki Y, Furuno M, Kasukawa T, Adachi J, Bono H, Kondo S, et al. Analysis of the mouse transcriptome based on functional annotation of 60,770 full-length cDNAs. Nature. 2002;420(6915):563-73. https://doi. org/10.1038/nature01266.

4. Kaikkonen MU, Adelman K. Emerging roles of non-coding RNA transcription. Trends Biochem Sci. 2018;43(9):654-67. https://doi.org/10.1016/j. tibs.2018.06.002.

5. Lin WY, Zhou QY, Wang CQ, Zhu LY, Bi C, Zhang SZ, et al. LncRNAs regulate metabolism in cancer. Int J Biol Sci. 2020;16(7):1194-206. https://doi. org/10.7150/ijbs.40769.

6. Bhan A, Soleimani M, Mandal SS. Long noncoding RNA and cancer: a new paradigm. Cancer Res. 2017;77(15):3965-81. https://doi. org/10.1158/0008-5472.CAN-16-2634.

7. Yu YL, Gao FB, He Q, Li GH, Ding GQ. LncRNA UCA1 functions as a ceRNA to promote prostate cancer progression via Sponging miR143. Mol Ther Nucleic Acid. 2020;19:751-8. https://doi.org/10.1016/j.omtn.2019.11.021.

8. Yan H, Li H, Li PY, Li X, Lin JJ, Zhu LL, et al. Long noncoding RNA MLK7-AS1 promotes ovarian cancer cells progression by modulating miR-375/YAP1 axis. J Exp Clin Cancer Res. 2018;37(1):1-18. https://doi.org/10.1186/s1304 6-018-0910-4

9. Xia WF, Liu Y, Cheng T, Xu T, Dong ML, Hu XP. Down-regulated IncRNA SBF2-AS1 inhibits tumorigenesis and progression of breast cancer by sponging microRNA-143 and repressing RRS1. J Exp Clin Cancer Res. 2020. https://doi.org/10.1016/10.1186/s13046-020-1520-5.

10. Ba MC, Ba Z, Long H, Cui SZ, Gong YF, Yan ZF, et al. LncRNA AC093818.1 accelerates gastric cancer metastasis by epigenetically promoting PDK1 expression. Cell Death Dis. 2020;11(1):64. https://doi.org/10.1038/s4141 9-020-2245-2.

11. Smolle MA, Bullock MD, Ling H, Pichler M, Haybaeck J. Long non-coding RNAs in endometrial carcinoma. Int J Mol Sci. 2015;16(11):26463-72. https://doi.org/10.3390/ijms161125962.
12. Li QL, Zhang C, Chen RC, Xiong HZ, Qiu FM, Liu SY, et al. Disrupting MALAT1/miR-200c sponge decreases invasion and migration in endometrioid endometrial carcinoma. Cancer Lett. 2016;383(1):28-40. https://doi. org/10.1016/j.canlet.2016.09.019.

13. Zhang YX, Hu P, Xie YQ, Kang YJ, Li M. IncRNA HOTAIR promotes endometrial carcinoma cells proliferation by binding to PTEN via activating PI3k/Akt signaling pathway. Mol Cell Biol. 2019;39(23):e00251. https://doi. org/10.1128/MCB.00251-19.

14. Dong PX, Xiong Y, Yue JM, Xu DZ, Ihira K, Konno Y, et al. Long noncoding RNA NEAT1 drives aggressive endometrial cancer progression via miR-361-regulated networks involving STAT3 and tumor microenvironment-related genes. J Exp Clin Cancer Res. 2019;38(1):295. https://doi. org/10.1186/s13046-019-1306-9.

15. Sun KX, Wu DD, Chen S, Zhao Y, Zong ZH. LncRNA MEG3 inhibit endometrial carcinoma tumorigenesis and progression through $\mathrm{PI} 3 \mathrm{~K}$ pathway. Apoptosis. 2017;22(12):1543-52. https://doi.org/10.1007/s1049 5-017-1426-7.

16. Kong Y, Ren Z. Overexpression of LnCRNA FER1L4 in endometrial carcinoma is associated with favorable survival outcome. Eur Rev Med Pharmacol Sci. 2018;22(23):8113-8. https://doi.org/10.26355/eurrev_20181 2_16502.

17. Wu SM, Liu H, Huang PJ, Chang IY, Lee CC, Yang CY, et al. circlncRNAnet: an integrated web-based resource for mapping functional networks of long or circular forms ofnoncoding RNAs. Gigascience. 2018;7(1):1-10. https://doi.org/10.1093/gigascience/gix118.

18. Tang ZF, Kang BX, Li CW, Chen TX, Zhang ZM. GEPIA2: an enhanced web server for large-scale expression profiling and interactive analysis. Nucleic Acids Res. 2019;47(1):556-60. https://doi.org/10.1093/nar/gkz430.

19. Nagy Á, Lánczky A, Menyhárt O, Gyorffy B. Validation of miRNA prognostic power in hepatocellular carcinoma using expression data of independent datasets. Sci Rep. 2018;8(1):1-9. https://doi.org/10.1038/s41598-01827521-y.

20. Casper J, Zweig AS, Villarreal C, Tyner C, Speir ML, Rosenbloom KR, et al. The UCSC genome browser database: 2018 update. Nucleic Acids Res. 2018;46(1):762-9. https://doi.org/10.1093/nar/gkx1020.

21. Volders PJ, Anckaert J, Verheggen K, Nuytens J, Martens L, Mestdagh P, et al. LNCipedia 5: towards a reference set of human long non-coding RNAs. Nucleic Acids Res. 2019;47(1):135-9. https://doi.org/10.1093/nar/ gky1031.

22. Cao Z, Pan X, Yang Y, Huang Y, Shen HB. The IncLocator: a subcellular localization predictor for long non-coding RNAs based on a stacked ensemble classifier. Bioinformatics. 2018;34(13):2185-94. https://doi. org/10.1093/bioinformatics/bty085.

23. Hou M, Tang X, Tian F, Shi FY, Liu FL, Gao G. AnnoLnc: a web server for systematically annotating novel human IncRNAs. BMC Genomics. 2016;17(1):931. https://doi.org/10.1186/s12864-016-3287-9.

24. Li JH, Liu S, Zhou H, Qu LH, Yang JH. starBase v2.0: decoding miRNAceRNA, miRNA-nCRNA and protein-RNA interaction networks from large-scale CLIP-Seq data. Nucleic Acids Res. 2014;42:D92-7. https://doi. org/10.1093/nar/gkt1248

25. Warde-Farley D, Donaldson SL, Comes O, Zuberi K, Badrawi R, Chao P, et al. The GeneMANIA prediction server: Biological network integration for gene prioritization and predicting gene function. Nucleic Acids Res. 2010;38(SUPPL.2):214-20. https://doi.org/10.1093/nar/gkq537.

26. Chou CH, Shrestha S, Yang CD, Chang NW, Lin YL, Liao KW, et al. miRTarBase update 2018: a resource for experimentally validated microRNAtarget interactions. Nucleic Acids Res. 2018;46(D1):D296-302. https://doi. org/10.1093/nar/gkx1067.

27. Shannon P, Markiel A, Ozier O, Baliga NS, Wang JT, Ramage D, et al. Cytoscape: a software environment for integrated models of biomolecular interaction networks. Genome Res. 2003;13(11):2498-504. https://doi. org/10.1101/gr.1239303.

28. Zhou YY, Zhou B, Pache L, Chang M, Khodabakhshi AH, Tanaseichuk O, et al. Metascape provides a biologist-oriented resource for the analysis of systems-level datasets. Nat Commun. 2019;10(1):1523. https://doi. org/10.1038/s41467-019-09234-6.

29. Li YS, Jiang TF, Zhou WW, Li JY, Li XH, Wang Q, et al. Pan-cancer characterization of immune-related IncRNAs identifies potential oncogenic biomarkers. Nat Commun. 2020;11(1):1000. https://doi.org/10.1038/s4146 7-020-14802-2. 
30. Spizzo R, Almeida MI, Colombatti A, Calin GA. Long non-coding RNAs and cancer: a new frontier of translational research. Oncogene. 2012;31(43):4577-87. https://doi.org/10.1038/onc.2011.621.

31. Kitagawa M, Kitagawa K, Kotake Y, Niida H, Ohhata T. Cell cycle regulation by long non-coding RNAs. Cell Mol Life Sci. 2013;70(24):4785-94. https:// doi.org/10.1007/s00018-013-1423-0.

32. Guo YJ, Bai MJ, Lin LT, Huang JJ, An YC, Liang LC, et al. LncRNA DLEU2 aggravates the progression of hepatocellular carcinoma through binding to EZH2. Biomed Pharmacother. 2019;118:109272. https://doi. org/10.1016/j.biopha.2019.109272.

33. Wang ZQ, Cai Q, Hu L, He CY, Li JF, Quan ZW, et al. Long noncoding RNA UCA 1 induced by SP1 promotes cell proliferation via recruiting EZH2 and activating AKT pathway in gastric cancer. Cell Death Dis. 2017;8(6):e2839. https://doi.org/10.1038/cddis.2017.143.

34. Wei S, Liu JH, Li X, Liu XY. Repression of IncRNA-SVUGP2 mediated by $\mathrm{EZH} 2$ contributes to the development of non-small cell lung cancer via brisking Wnt/ $\beta$-catenin signal. Artif Cells Nanomedicine Biotechnol. 2019;47(1):3400-9. https://doi.org/10.1080/21691401.2019.1648279.

35. Guttman M, Rinn JL. Modular regulatory principles of large non-coding RNAs. Nature. 2014;482(7385):339-46. https://doi.org/10.1038/natur e10887.

36. Salmena L, Poliseno L, Tay Y, Kats L, Pandolfi PP, Pandolfi PP. Essay a ceRNA hypothesis : the rosetta stone of a hidden RNA language? Cell. 2011;146(3):353-8. https://doi.org/10.1016/j.cell.2011.07.014.

37. Qu L, Ding J, Chen C, Sun YH, Wang HY, et al. Exosome-transmitted IncARSR promotes sunitinib resistance in renal cancer by acting as a competing endogenous RNA. Cancer Cell. 2016;29(5):653-68. https://doi. org/10.1016/j.ccell.2016.03.004.

38. Xiao GA, Yao JJ, Kong DP, Ye C, Chen R, Li L, et al. The long noncoding RNA TTTY15, which is located on the y chromosome, promotes prostate cancer progression by sponging let-7. Eur Urol. 2018;76(3):315-26. https ://doi.org/10.1016/j.eururo.2018.11.012.

39. Zhou YX, Wang C, Mao LW, Wang YL, Xia LQ, Zhao WL, et al. Long noncoding RNA HOTAIR mediates the estrogen-induced metastasis of endometrial cancer cells via the miR-646/NPM1 axis. Am J Physiol Cell Physiol. 2018;314(6):690-701. https://doi.org/10.1152/ajpcell.00222.2017.

40. Wu XL, Cai DG, Zhang F, Li M, Wan QY. Long noncoding RNA TUSC7 inhibits cell proliferation, migration and invasion by regulating SOCS4 (SOCS5) expression through targeting miR- 616 in endometrial carcinoma. Life Sci. 2019;231:116549. https://doi.org/10.1016/j.lfs.2019.116549.

41. Liu L, Hu JG, Yu TH, You S, Zhang YL, Hu LN. miR-27b-3p/MARCH7 regulates invasion and metastasis of endometrial cancer cells through Snail-mediated pathway. Acta Biochim Biophys Sin. 2019;51(5):492-500. https://doi.org/10.1093/abbs/gmz030.

42. Bao W, Wang HH, Tian FJ, He XY, Qiu MT, Wang JY, et al. A TrkB-STAT3miR-204-5p regulatory circuitry controls proliferation and invasion of endometrial carcinoma cells. Mol Cancer. 2013;12:155. https://doi. org/10.1186/1476-4598-12-155.
43. Zhang XM, Dong Y, Ti HJ, Zhao L, Wang Y, Li T, et al. Down-regulation of miR-145 and miR-143 might be associated with DNA methyltransferase 3B overexpression and worse prognosis in endometrioid carcinomas. Hum Pathol. 2013;44(11):2571-80. https://doi.org/10.1016/j.humpa th.2013.07.002

44. Xu YY, Wu HJ, Ma HD, Xu LP, Huo Y, Yin LR. MicroRNA-503 suppresses proliferation and cell cycle progression of endometrioid endometrial cancer via negatively regulating cyclin D1. FEBS J. 2013;280(16):3768-79. https:// doi.org/10.1111/febs.12365.

45. Jiang HY, Lie Y, Li J, Zhang XY, Niu G, Chen SQ, et al. Long noncoding RNA LSINCT5 promotes endometrial carcinoma cell proliferation, cycle, and invasion by promoting the Wnt/ $\beta$-catenin signaling pathway via HMGA2. Ther Adv Med Oncol. 2019;11:1-13. https://doi.org/10.1177/1758835919 874649.

46. LVYQ, Chen SR, Wu JJ, Lin RY, Zhou LM, Chen GM, et al. Upregulation of long non-coding RNA OGFRP1 facilitates endometrial cancer by regulating miR-124-3p/SIRT1 axis and by activating PI3K/AKT/GSK-3 $\beta$ pathway. Artif Cells Nanomed Biotechnol. 2019;47(1):2083-90. https:// doi.org/10.1080/21691401.2019.1617727.

47. Liu HY, Wan JH, Chu J. Long non-coding RNAs and endometrial cancer. Biomed Pharmacother. 2019;119:109396. https://doi.org/10.1016/j.bioph a.2019.109396.

48. Crumley S, Kurnit K, Hudgens C, Fellman B, Tetzlaff MT, Broaddus R. Identification of a subset of microsatellite-stable endometrial carcinoma with high PD-L1 and CD8 + lymphocytes. Mod Pathol. 2019;32(3):396-404. https://doi.org/10.1038/s41379-018-0148-x.

49. Brown DM. Cytolytic CD4 cells: direct mediators in infectious disease and malignancy. Cell Immunol. 2010;262(2):89-95. https://doi.org/10.1016/j. cellimm.2010.02.008.

50. Bellone S, Centritto F, Black J, Schwab C, English D, Cocco E, et al. Polymerase epsilon (POLE) ultra-mutated tumors induce robust tumorspecific CD4 + T cell responses in endometrial cancer patients. Gynecol Oncol. 2015;138(1):11-7. https://doi.org/10.1016/j.ygyno.2015.04.027.

51. Gentles AJ, Newman AM, Liu CL, Bratman SV, Feng WG, Kim D, et al. The prognostic landscape of genes and infiltrating immune cells across human cancers. Nat Med. 2015;21(8):938-45. https://doi.org/10.1038/ $\mathrm{nm} .3909$.

52. Blaisdell A, Crequer A, Columbus D, Daikoku T, Mittal K, Dey SK, et al. Neutrophils oppose uterine epithelial carcinogenesis via debridement of hypoxic tumor cells. Cancer Cell. 2015;28(6):785-99. https://doi. org/10.1016/j.ccell.2015.11.005.

\section{Publisher's Note}

Springer Nature remains neutral with regard to jurisdictional claims in published maps and institutional affiliations.
Ready to submit your research? Choose BMC and benefit from:

- fast, convenient online submission

- thorough peer review by experienced researchers in your field

- rapid publication on acceptance

- support for research data, including large and complex data types

- gold Open Access which fosters wider collaboration and increased citations

- maximum visibility for your research: over 100M website views per year

At BMC, research is always in progress.

Learn more biomedcentral.com/submissions 